\title{
Birds of a Feather? Magellan/IMACS Spectroscopy of the Ultra-faint Satellites Grus II, Tucana IV, and Tucana $\mathrm{V}^{*}$
}

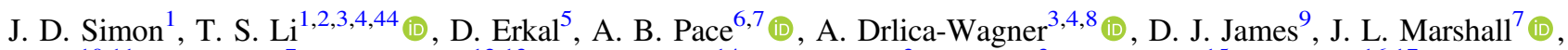

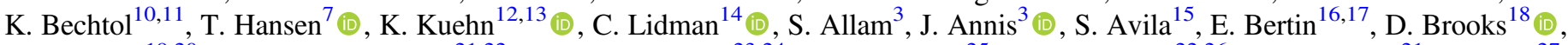
D. L. Burke ${ }^{19,20}$ (1) A. Carnero Rosell ${ }^{21,22}$, M. Carrasco Kind ${ }^{23,24}$ (i) , J. Carretero ${ }^{25}$, L. N. da Costa ${ }^{22,26}$, J. De Vicente ${ }^{21}$, S. Desai $^{27}$,

P. Doel ${ }^{18}$, T. F. Eifler ${ }^{28,29}$, S. Everett ${ }^{30}$, P. Fosalba ${ }^{31,32}$, J. Frieman ${ }^{3,4}$, J. García-Bellido ${ }^{15}$ (i), E. Gaztanaga ${ }^{31,32}$,

D. W. Gerdes ${ }^{33,34}$ (1), D. Gruen ${ }^{19,20,35}$ (1), R. A. Gruendl ${ }^{23,24}$ (1) J. Gschwend ${ }^{22,26}$, G. Gutierrez ${ }^{3}$, D. L. Hollowood ${ }^{30}$ (1),

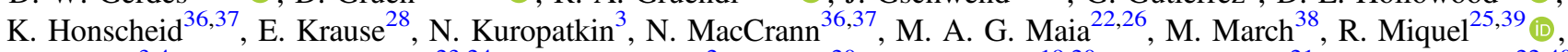

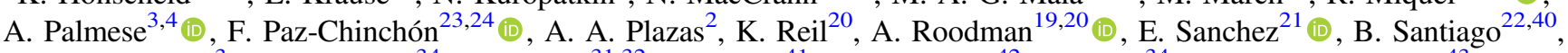

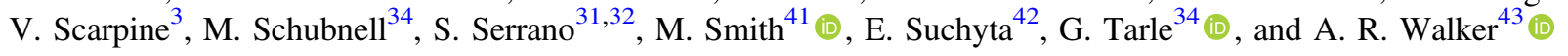
(DES Collaboration)

\footnotetext{
${ }^{1}$ Observatories of the Carnegie Institution for Science, 813 Santa Barbara St., Pasadena, CA 91101, USA

${ }^{2}$ Department of Astrophysical Sciences, Princeton University, Peyton Hall, Princeton, NJ 08544, USA

${ }^{3}$ Fermi National Accelerator Laboratory, P.O. Box 500, Batavia, IL 60510, USA

${ }^{4}$ Kavli Institute for Cosmological Physics, University of Chicago, Chicago, IL 60637, USA

${ }^{5}$ Department of Physics, University of Surrey, Guildford GU2 7XH, UK

${ }^{6}$ Department of Physics, Carnegie Mellon University, Pittsburgh, PA 15312, USA

${ }^{7}$ George P. and Cynthia Woods Mitchell Institute for Fundamental Physics and Astronomy, and Department of Physics and Astronomy, Texas A\&M University, College Station, TX 77843, USA

${ }_{9}^{8}$ Department of Astronomy and Astrophysics, University of Chicago, Chicago, IL 60637, USA

${ }^{9}$ Center for Astrophysics | Harvard \& Smithsonian, 60 Garden St., Cambridge, MA 02138, USA ${ }^{10}$ LSST, 933 North Cherry Ave., Tucson, AZ 85721, USA

${ }^{11}$ Physics Department, 2320 Chamberlin Hall, University of Wisconsin-Madison, 1150 University Ave., Madison, WI 53706-1390, USA

${ }^{12}$ Australian Astronomical Optics, Macquarie University, North Ryde, NSW 2113, Australia

${ }^{13}$ Lowell Observatory, 1400 Mars Hill Rd., Flagstaff, AZ 86001, USA

${ }^{14}$ The Research School of Astronomy and Astrophysics, Australian National University, ACT 2601, Australia

${ }^{15}$ Instituto de Fisica Teorica UAM/CSIC, Universidad Autonoma de Madrid, E-28049 Madrid, Spain

${ }^{16}$ CNRS, UMR 7095, Institut d'Astrophysique de Paris, F-75014, Paris, France

${ }^{17}$ Sorbonne Universités, UPMC Univ Paris 06, UMR 7095, Institut d'Astrophysique de Paris, F-75014, Paris, France

${ }^{18}$ Department of Physics \& Astronomy, University College London, Gower St., London, WC1E 6BT, UK

${ }^{19}$ Kavli Institute for Particle Astrophysics \& Cosmology, P.O. Box 2450, Stanford University, Stanford, CA 94305, USA

${ }^{20}$ SLAC National Accelerator Laboratory, Menlo Park, CA 94025, USA

${ }^{21}$ Centro de Investigaciones Energéticas, Medioambientales y Tecnológicas (CIEMAT), Madrid, Spain

${ }^{22}$ Laboratório Interinstitucional de e-Astronomia-LIneA, Rua Gal. José Cristino 77, Rio de Janeiro, RJ-20921-400, Brazil

${ }^{23}$ Department of Astronomy, University of Illinois at Urbana-Champaign, 1002 W. Green St., Urbana, IL 61801, USA

${ }^{24}$ National Center for Supercomputing Applications, 1205 West Clark St., Urbana, IL 61801, USA

${ }^{25}$ Institut de Física d'Altes Energies (IFAE), The Barcelona Institute of Science and Technology, Campus UAB, E-08193 Bellaterra (Barcelona) Spain

${ }^{26}$ Observatório Nacional, Rua Gal. José Cristino 77, Rio de Janeiro, RJ-20921-400, Brazil

${ }^{28}$ Department of Astronomy/Steward Observatory, University of Arizona, 933 North Cherry Ave., Tucson, AZ 85721-0065, USA

${ }^{29}$ Jet Propulsion Laboratory, California Institute of Technology, 4800 Oak Grove Dr., Pasadena, CA 91109, USA

${ }^{30}$ Santa Cruz Institute for Particle Physics, Santa Cruz, CA 95064, USA

${ }^{31}$ Institut d'Estudis Espacials de Catalunya (IEEC), E-08034 Barcelona, Spain

${ }^{32}$ Institute of Space Sciences (ICE, CSIC), Campus UAB, Carrer de Can Magrans, s/n, E-08193 Barcelona, Spain

${ }_{3}$ Department of Astronomy, University of Michigan, Ann Arbor, MI 48109, USA

${ }^{34}$ Department of Physics, University of Michigan, Ann Arbor, MI 48109, USA

35 Department of Physics, Stanford University, 382 Via Pueblo Mall, Stanford, CA 94305, USA

${ }^{36}$ Center for Cosmology and Astro-Particle Physics, The Ohio State University, Columbus, OH 43210, USA

${ }^{37}$ Department of Physics, The Ohio State University, Columbus, OH 43210, USA

${ }^{38}$ Department of Physics and Astronomy, University of Pennsylvania, Philadelphia, PA 19104, USA

${ }^{39}$ Institució Catalana de Recerca i Estudis Avançats, E-08010 Barcelona, Spain

${ }^{40}$ Instituto de Física, UFRGS, Caixa Postal 15051, Porto Alegre, RS-91501-970, Brazil

${ }^{41}$ School of Physics and Astronomy, University of Southampton, Southampton, SO17 1BJ, UK

${ }^{42}$ Computer Science and Mathematics Division, Oak Ridge National Laboratory, Oak Ridge, TN 37831, USA

${ }^{43}$ Cerro Tololo Inter-American Observatory, National Optical Astronomy Observatory, Casilla 603, La Serena, Chile

Received 2019 November 19; revised 2020 January 27; accepted 2020 February 14; published 2020 April 7
}

\begin{abstract}
We present Magellan/IMACS spectroscopy of three recently discovered ultra-faint Milky Way satellites, Grus II, Tucana IV, and Tucana V. We measure systemic velocities of $v_{\text {hel }}=-110.0 \pm 0.5 \mathrm{~km} \mathrm{~s}^{-1}, v_{\text {hel }}=15.9_{-1.7}^{+1.8}$ $\mathrm{km} \mathrm{s}^{-1}$, and $v_{\text {hel }}=-36.2_{-2.2}^{+2.5} \mathrm{~km} \mathrm{~s}^{-1}$ for the three objects, respectively. Their large relative velocities demonstrate
\end{abstract}

\footnotetext{
* This paper includes data gathered with the $6.5 \mathrm{~m}$ Magellan Telescopes located at Las Campanas Observatory, Chile.

${ }^{44}$ NHFP Einstein Fellow.
} 
that the satellites are unrelated despite their close physical proximity. We determine a velocity dispersion for Tuc IV of $\sigma=4.3_{-1.0}^{+1.7} \mathrm{~km} \mathrm{~s}^{-1}$, but we cannot resolve the velocity dispersions of the other two systems. For Gru II, we place an upper limit ( $90 \%$ confidence) on the dispersion of $\sigma<1.9 \mathrm{~km} \mathrm{~s}^{-1}$, and for Tuc V, we do not obtain any useful limits. All three satellites have metallicities below $[\mathrm{Fe} / \mathrm{H}]=-2.1$, but none has a detectable metallicity spread. We determine proper motions for each satellite based on Gaia astrometry and compute their orbits around the Milky Way. Gru II is on a tightly bound orbit with a pericenter of $25_{-7}^{+6} \mathrm{kpc}$ and orbital eccentricity of $0.45_{-0.05}^{+0.08}$. Tuc V likely has an apocenter beyond $100 \mathrm{kpc}$ and could be approaching the Milky Way for the first time. The current orbit of Tuc IV is similar to that of Gru II, with a pericenter of $25_{-8}^{+11} \mathrm{kpc}$ and an eccentricity of $0.36_{-0.06}^{+0.13}$. However, a backward integration of the position of Tuc IV demonstrates that it collided with the Large Magellanic Cloud at an impact parameter of $4 \mathrm{kpc} \sim 120 \mathrm{Myr}$ ago, deflecting its trajectory and possibly altering its internal kinematics. Based on their sizes, masses, and metallicities, we classify Gru II and Tuc IV as likely dwarf galaxies, but the nature of Tuc $\mathrm{V}$ remains uncertain.

Unified Astronomy Thesaurus concepts: Local Group (929); Dwarf galaxies (416); Magellanic Clouds (990); Milky Way Galaxy (1054); Dark matter (353)

Supporting material: machine-readable tables

\section{Introduction}

Over the past four years, analyses of the first deep, wide-field digital surveys of the southern sky have significantly expanded the population of Milky Way satellites and pushed the search for faint dwarf galaxies to lower surface brightnesses than was previously possible (Bechtol et al. 2015; Drlica-Wagner et al. 2015, 2016; Kim et al. 2015; Kim \& Jerjen 2015; Koposov et al. 2015, 2018; Laevens et al. 2015a, 2015b; Torrealba et al. 2016a, 2016b, 2018). Motivated by the apparent concentration of satellites near the Large and Small Magellanic Clouds, one particularly active area of recent study is the association of dwarf galaxies with the Magellanic system (e.g., Sales et al. 2011; Deason et al. 2015; Jethwa et al. 2016; Sales et al. 2017; Kallivayalil et al. 2018; Erkal \& Belokurov 2019; Pardy et al. 2019). Dwarfs may also be associated with each other in even smaller groups (e.g., Li \& Helmi 2008; Klimentowski et al. 2010).

Several of the recently discovered Milky Way satellites are in very close physical proximity to each other, sparking the suggestion that they could represent bound (or formerly bound) associations of dwarfs. Carina II and Carina III are just $8 \mathrm{kpc}$ apart at present (Torrealba et al. 2018), but their radial velocities (Li et al. 2018b) and orbits (Simon 2018) are so different that this configuration appears to be simply a coincidence. Similarly, Drlica-Wagner et al. (2015) noted that among the satellites discovered by the Dark Energy Survey (DES; Diehl et al. 2014; Flaugher et al. 2015), Tucana II, Tucana IV (Tuc IV), and Tucana V (Tuc V) are all within $7 \mathrm{kpc}$ of a common centroid. Grus II (Gru II) is located less than $18 \mathrm{kpc}$ from this position as well. The radial velocity of Tucana II has been measured (Walker et al. 2016), but no spectroscopy is available for the other three systems, so the viability of their association with each other or with the Magellanic Clouds has not been tested. Finding dwarf galaxies that formed with only other dwarfs as their neighbors will enable novel tests of the effects of environment on early galaxy formation and evolution (e.g., D’Onghia \& Lake 2008; Wetzel et al. 2015; Fillingham et al. 2019; Rodriguez Wimberly et al. 2019).

This paper continues a series of publications aimed at characterizing recently discovered Milky Way satellites (Simon et al. 2015, 2017; Li et al. 2017, 2018b). Here we present the first stellar spectroscopy of Gru II, Tuc IV, and Tuc V and assess the nature of each object. In Section 2, we describe our observations and data reduction. We measure radial velocities and metallicities of the observed stars and identify members of the three satellites in Section 3. In Section 4, we calculate their dynamical masses, metallicity distributions, proper motions, and orbits around the Milky Way. We discuss the nature and origin of each satellite in Section 5 and summarize our findings in Section 6.

\section{Observations and Data Reduction}

\subsection{Spectrograph Setup and Summary of Observations}

We observed Gru II, Tuc IV, and Tuc V with the IMACS spectrograph (Dressler et al. 2006) on the Magellan/Baade telescope during a number of observing runs spanning from 2015 August to 2018 September. As in previous studies (Simon et al. 2017; Li et al. 2017, 2018b), we used the $f / 4$ camera and the $1200 \mathrm{lmm}^{-1}$ grating blazed at $9000 \AA$ to produce $R \approx 11,000$ spectra from $\sim 7500$ to $8900 \AA$. We observed seven slit masks targeting Gru II, nine slit masks targeting Tuc IV, and one slit mask targeting Tuc V. Each mask was designed with 0 " $7 \times 5^{\prime \prime}$ slitlets. Typically, our observing sequence consisted of several science exposures totaling 1-2 hr followed by comparison lamp and flat-field frames. The comparison lamps used were Ne, Ar, and He through 2015 October; Ne, Ar, and Kr from 2015 November until 2016 August; and Ne, Ar, Kr, and He beginning in 2017. Total integration times for each slit mask ranged from 0.5 to $\sim 10 \mathrm{hr}$. A summary of the observing dates and mask parameters is provided in Table 1.

\subsection{Target Selection}

Spectroscopic targets were selected from the DES Y2Q1 photometric catalog. The target selection criteria were identical to those used by Simon et al. (2017) for Tucana III. Candidate red giant branch (RGB) stars were selected in a window defined by a $12 \mathrm{Gyr},[\mathrm{Fe} / \mathrm{H}]=-2.20$ PARSEC isochrone (Bressan et al. 2012) on the red side and the An et al. (2008) M92 fiducial sequence (transformed from the Sloan Digital Sky Survey to the DES photometric system) on the blue side. ${ }^{45}$ Stars outside this window but within 0.03 mag of either edge were targeted at reduced priority. Candidate horizontal branch stars in these systems are located in a relatively clean part of the color-magnitude diagram (CMD) and were selected according

\footnotetext{
45 Note that the PARSEC isochrones relied on outdated throughput curves for the DES filters at the time of the target selection for this paper. This problem was corrected in 2017 July (see appendix of Li et al. 2018b), so isochrones downloaded now will not match those used for our target selection. Nevertheless, because our color selection window was defined to be wider than the giant branch of known ultra-faint dwarfs, use of the old isochrones should not bias our spectroscopic target selection.
} 
Table 1

Summary of IMACS Spectroscopic Observations

\begin{tabular}{|c|c|c|c|c|c|c|c|}
\hline $\begin{array}{l}\text { Mask } \\
\text { name }\end{array}$ & $\begin{array}{l}\alpha \\
\alpha(\mathrm{J} 2000) \\
\left(\begin{array}{lll}\mathrm{h} & \mathrm{m} & \mathrm{s}\end{array}\right)\end{array}$ & 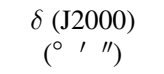 & $\begin{array}{l}\text { Slit PA } \\
\text { (deg) }\end{array}$ & $\begin{array}{l}t_{\exp } \\
(\mathrm{s})\end{array}$ & $\begin{array}{c}\text { MJD of } \\
\text { observation }^{\mathrm{a}}\end{array}$ & \# of slits & $\begin{array}{c}\% \text { useful } \\
\text { spectra }\end{array}$ \\
\hline \multirow{2}{*}{$\begin{array}{l}\text { Gru II Mask } 1 \\
\ldots\end{array}$} & 220346.80 & -462850.0 & 240.0 & 20400 & 57282.59 & 75 & $41 \%$ \\
\hline & $\cdots$ & $\cdots$ & $\cdots$ & 7200 & 57634.36 & $\cdots$ & $20 \%$ \\
\hline \multirow{2}{*}{$\begin{array}{l}\text { Gru II Mask } 3 \\
\ldots\end{array}$} & 220400.00 & -461900.0 & 300.0 & 7200 & 57284.08 & 59 & $42 \%$ \\
\hline & $\ldots$ & $\ldots$ & $\ldots$ & 5400 & 57630.53 & $\ldots$ & $42 \%$ \\
\hline Gru II Mask 4 & 220357.00 & -462747.0 & 260.0 & 34800 & 57980.89 & 67 & $45 \%$ \\
\hline Gru II Mask 7 & 220337.00 & -462725.0 & 134.0 & 7920 & 58363.60 & 50 & $40 \%$ \\
\hline \multirow{3}{*}{$\begin{array}{l}\text { Tuc IV Mask } 1 \\
\ldots \\
\ldots\end{array}$} & 000302.20 & -604910.0 & 226.0 & 9600 & 57247.35 & 58 & $45 \%$ \\
\hline & $\ldots$ & ... & $\ldots$ & 9600 & 57282.32 & $\ldots$ & $47 \%$ \\
\hline & $\ldots$ & $\ldots$ & $\ldots$ & 9600 & 57346.11 & $\ldots$ & $52 \%$ \\
\hline Tuc IV Mask 2 & 000244.00 & -604927.0 & 166.2 & 15480 & 57633.91 & 66 & $26 \%$ \\
\hline Tuc IV Mask 3 & 000151.00 & -605530.0 & 154.0 & 4800 & 57981.23 & 44 & $34 \%$ \\
\hline Tuc IV Mask 8 & 000259.00 & -610900.0 & 145.0 & 1800 & 58340.13 & 5 & $40 \%$ \\
\hline Tuc IV Mask 9 & 000213.00 & -604615.0 & 134.0 & 9600 & 58363.75 & 8 & $63 \%$ \\
\hline \multirow[t]{3}{*}{ Tuc V Mask 1} & 233740.00 & -631634.0 & 246.0 & 3840 & 57283.32 & 65 & $17 \%$ \\
\hline & $\ldots$ & $\ldots$ & $\ldots$ & 9600 & 57312.23 & $\cdots$ & $23 \%$ \\
\hline & $\ldots$ & $\ldots$ & $\ldots$ & 33840 & 57568.70 & $\ldots$ & $43 \%$ \\
\hline
\end{tabular}

Note.

${ }^{\text {a }}$ For observations made over multiple nights, the date listed here is the weighted mean observation date, which may occur during daylight hours.

to the photometric membership probabilities from DrlicaWagner et al. (2015). We also targeted main sequence turnoff stars based on their membership probabilities.

In 2016 July, we obtained some shallow spectroscopy of wide fields surrounding Gru II and Tuc IV with the AAOmega spectrograph (Sharp et al. 2006) on the Anglo-Australian Telescope (AAT). These observations took place in poor conditions and served primarily to measure the velocities of bright stars to see if any could be associated with Gru II or Tuc IV. We identified one new member of Gru II in this data set (which we subsequently confirmed with IMACS) and eliminated many stars in the photometric selection region from further consideration. Targeting for the 2017 and 2018 IMACS observations (Gru II Masks 4-7 and Tuc IV Masks 4-9) employed this information. Although the AAT velocity measurements are not used in our analysis, we provide the data in Table 2 for the benefit of other researchers interested in these fields.

For the 2018 IMACS observations (Gru II Masks 6-7 and Tuc IV Masks 5-9), we also included astrometry from the second Gaia data release (DR2; Gaia Collaboration et al. 2018) as an additional (loose) selection criterion. Stars with parallaxes differing from zero at less than $2 \sigma$ significance and with proper motions within $3 \sigma$ of the mean proper motion established by the member stars we had already identified to that point were considered candidate members. The color-magnitude and spatial distributions of target stars are displayed in the left and middle panels, respectively, of Figures 1-3.

\subsection{Data Reduction}

We reduced the IMACS observations using the procedures described by Simon et al. (2017) and Li et al. (2017). Briefly, we used the Cosmos reduction pipeline (Dressler et al. 2011; Oemler et al. 2017) to create a map of the slits across the detector mosaic and generate a preliminary wavelength solution. We then employed the IMACS reduction pipeline developed by Simon et al. (2017), which is based on the DEEP2 data reduction pipeline (Cooper et al. 2012; Newman et al. 2013), to calibrate and extract one-dimensional spectra from the raw two-dimensional data. We note that the IMACS detector arrays were swapped between the $f / 2$ and $f / 4$ cameras in 2017 December. The CCD mosaic used in $f / 4$ beginning in 2018 has slightly higher efficiency at the wavelength of our observations, at the cost of increased cosmetic artifacts and fringing. The artifacts can be removed by slightly changing the grating angle between sets of observations, and the fringing can be removed via flat-field frames.

\section{Velocity and Metallicity Measurements}

\subsection{Radial Velocity Measurements}

We measure radial velocities from the reduced IMACS spectra following the procedures described by Simon et al. (2017) and Li et al. (2017). We compute $\chi^{2}$ for each spectrum as a function of velocity relative to a template spectrum of a bright metal-poor star. The template spectra were obtained with IMACS using the same spectrograph setup but driving the star across the spectrograph slit during the exposure in order to 
Table 2

AAT Velocity Measurements for Gru II and Tuc IV

\begin{tabular}{|c|c|c|c|c|c|c|c|}
\hline ID & MJD & $\begin{array}{l}\text { R.A. } \\
\text { (deg) }\end{array}$ & $\begin{array}{l}\text { Decl. } \\
\text { (deg) }\end{array}$ & $\begin{array}{c}g^{\mathrm{a}} \\
(\mathrm{mag})\end{array}$ & $\begin{array}{c}r^{\mathrm{a}} \\
(\mathrm{mag})\end{array}$ & $\mathrm{S} / \mathrm{N}$ & $\begin{array}{c}v \\
\left(\mathrm{~km} \mathrm{~s}^{-1}\right)\end{array}$ \\
\hline DES J215828.76-463329.6 & 57596.1 & 329.61985 & -46.55823 & 17.73 & 17.03 & 6.8 & $4.8 \pm 3.5$ \\
\hline DES J215831.30-462702.6 & 57596.1 & 329.63040 & -46.45072 & 17.22 & 16.43 & 17.8 & $-11.4 \pm 2.2$ \\
\hline DES J215851.85-464030.5 & 57596.1 & 329.71605 & -46.67514 & 17.90 & 17.23 & 7.3 & $-62.9 \pm 3.3$ \\
\hline DES J215857.30-460420.0 & 57596.1 & 329.73874 & -46.07222 & 17.06 & 16.13 & 29.0 & $-12.5 \pm 0.8$ \\
\hline DES J215903.05-465407.6 & 57596.1 & 329.76272 & -46.90211 & 17.06 & 16.23 & 8.6 & $-37.0 \pm 3.0$ \\
\hline DES J215909.17-463536.3 & 57596.1 & 329.78819 & -46.59340 & 17.78 & 17.11 & 9.0 & $-7.6 \pm 3.1$ \\
\hline DES J215915.51-461956.4 & 57596.1 & 329.81464 & -46.33234 & 17.10 & 16.28 & 26.5 & $-17.4 \pm 2.6$ \\
\hline DES J215917.67-465605.9 & 57596.1 & 329.82361 & -46.93498 & 17.31 & 16.56 & 9.8 & $41.2 \pm 3.9$ \\
\hline DES J215923.64-460638.6 & 57596.1 & 329.84849 & -46.11072 & 17.19 & 16.38 & 26.4 & $-16.1 \pm 1.5$ \\
\hline DES J215924.02-461309.8 & 57596.1 & 329.85008 & -46.21940 & 17.57 & 16.77 & 11.4 & $54.2 \pm 1.9$ \\
\hline
\end{tabular}

\section{Note.}

${ }^{a}$ Quoted magnitudes represent the weighted-average dereddened point-spread function (PSF) magnitude derived from the DES images using SourceExtractor (DrlicaWagner et al. 2015).

(This table is available in its entirety in machine-readable form.)

create a uniform slit illumination profile. For RGB candidates, we use HD 122563 as the template star, and we assume that its velocity is $v_{\text {hel }}=-26.51 \mathrm{~km} \mathrm{~s}^{-1}$ (Chubak et al. 2012). For horizontal branch candidates, we use HD 161817 as the template star; while the spectrum of this hot star is dominated by the hydrogen Paschen series lines, the $\mathrm{Ca}$ triplet (CaT) lines are visible as well, and we use them to tie the velocity of the template spectrum to that of $\mathrm{HD} 122563$. We measure a velocity of $v_{\text {hel }}=-363.27 \mathrm{~km} \mathrm{~s}^{-1}$ for HD 161817, identical within the uncertainties to the value of $v_{\text {hel }}=-363.2 \pm 0.4$ $\mathrm{km} \mathrm{s}^{-1}$ determined by Gontcharov (2006). We remove velocity offsets resulting from imperfect centering of each star in its slitlet using template fits of the $A$-band region of each spectrum to a spectrum of the hot, rapidly rotating star HR 4781.

As in previous papers, because of the inferior wavelength solutions obtained without the $\mathrm{Kr}$ comparison lamp, the systematic velocity uncertainty is $1.2 \mathrm{~km} \mathrm{~s}^{-1}$ for observations obtained through 2015 October and $1.0 \mathrm{~km} \mathrm{~s}^{-1}$ beginning in 2015 November. The statistical uncertainty on each velocity measurement is determined by adding normally distributed noise to the observed spectrum and remeasuring the velocity 500 times (Simon \& Geha 2007; Simon et al. 2017). The total velocity uncertainties consist of the quadrature sum of the statistical and systematic uncertainties.

\subsection{Metallicity Measurements}

We measure metallicities from the equivalent widths (EWs) of the CaT lines using the methods discussed in Simon et al. $(2015,2017)$ and Li et al. (2017). We fit the CaT lines using a Gaussian plus Lorentzian profile and integrate the fitted profile to determine the EW of each of the three lines. We assume a systematic uncertainty on EW measurements of $0.32 \AA$ (Simon et al. 2017), which we add in quadrature with the measurement uncertainty to obtain a total uncertainty. We convert the summed EWs to metallicity with the calibration of Carrera et al. (2013). Although Gru II and Tuc IV do contain a handful of HB stars, unlike Reticulum II and Tucana III, for consistency with past work (and with Tuc V, which lacks any HB stars), we continue to employ the metallicity calibration based on absolute $V$ magnitude. If we used the $V-V_{\mathrm{HB}}$ calibration from Carrera et al. (2013) instead, we would derive lower metallicities by $\sim 0.1$ dex on average.

\subsection{Spectroscopic Membership Determination}

For each satellite, we determine an approximate value for the systemic velocity by considering the most likely member candidates (stars lying along the fiducial sequences determined from previously studied DES dwarfs and closest to the center of the object) from the initial target selection. We then select stars within the various photometric selection regions and with velocities within $15 \mathrm{~km} \mathrm{~s}^{-1}$ of the systemic velocity as member candidates. The candidates are inspected individually, considering their precise position in the CMD, metallicity (if available), and other spectral diagnostics such as the Mg $\lambda$ $\lambda 8807 \AA$ line (Battaglia \& Starkenburg 2012). Finally, we cross-match the resulting set of stars with the Gaia DR2 catalog. As with the other ultra-faint satellites (Simon 2018), the member stars of Gru II and Tuc IV are tightly clustered in proper motion space (Massari \& Helmi 2018), and any outliers are immediately obvious (see Figure 4). One candidate Tuc IV member is rejected on the basis of Gaia astrometry. Because Tuc V only contains a single member candidate brighter than $g=20$, the Gaia proper motions do not provide as much discriminatory power, but we verify that the fainter candidates have proper motions consistent with the brighter one despite their large uncertainties. In particular, three of the five highest-priority Tuc $\mathrm{V}$ target stars are clustered at a velocity of $\sim-36 \mathrm{~km} \mathrm{~s}^{-1}$, confirming the detection of the satellite despite the small sample (see Figure 5).

After combining the available photometric data, radial velocities, metallicities, spectral diagnostics, and astrometry, most of the members of each system are unambiguous. The exceptions are one star each in Gru II (DES J220400.12-462529.0) and Tuc IV (DES J000311.46-604451.5), which resemble members in every way except that their color places them $\sim 0.1$ mag redder than the RGB at their magnitude. Conceivably, these stars could be members that are carbon-enhanced, similar to one star in Hydrus I identified by Koposov et al. (2018) and another star in Canes Venatici I studied by Zucker et al. (2006b) and Yoon et al. (2019). Additional spectroscopy at shorter wavelengths where carbon features are prominent would be needed to test this possibility. However, given the currently available data for these stars, we consider them nonmembers. In both cases, because their 

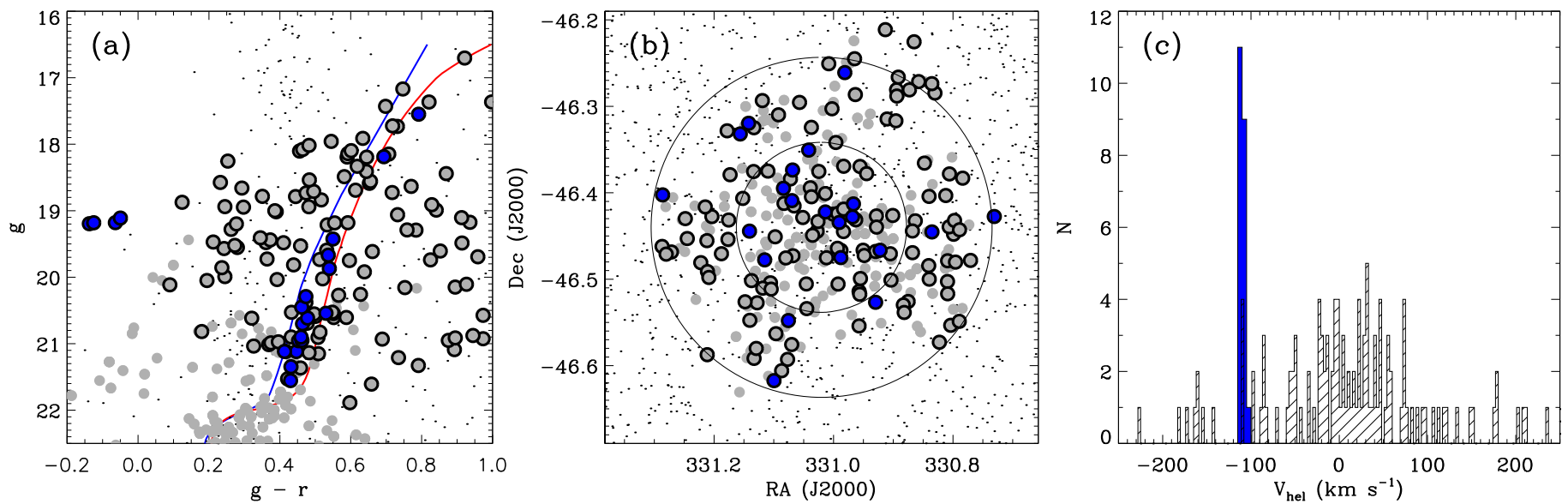

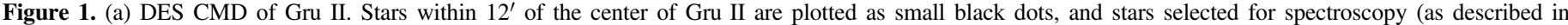

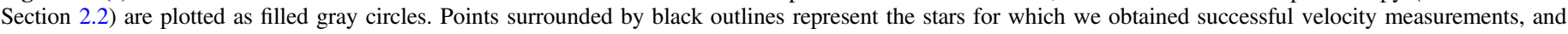

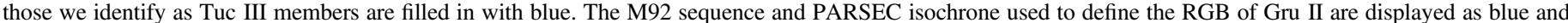

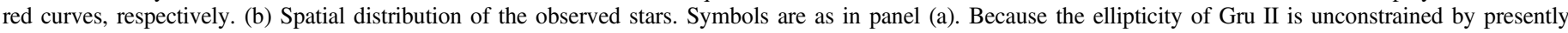

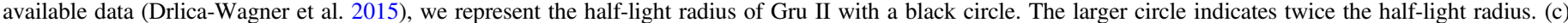

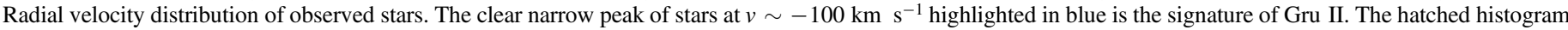
indicates stars that are not members of Gru II.

Table 3

IMACS Velocity and Metallicity Measurements for Gru II, Tuc IV, and Tuc V

\begin{tabular}{|c|c|c|c|c|c|c|c|c|c|c|}
\hline ID & $\begin{array}{l}\text { R.A. } \\
\text { (deg) }\end{array}$ & $\begin{array}{l}\text { Decl. } \\
\text { (deg) }\end{array}$ & $\begin{array}{c}g^{\mathrm{a}} \\
(\mathrm{mag})\end{array}$ & $\begin{array}{c}r^{\mathrm{a}} \\
(\mathrm{mag})\end{array}$ & MJD & $\mathrm{S} / \mathrm{N}$ & $\left(\begin{array}{c}v \\
\left(\mathrm{~km} \mathrm{~s}^{-1}\right)\end{array}\right.$ & $\begin{array}{l}\text { EW } \\
(\AA)\end{array}$ & {$[\mathrm{Fe} / \mathrm{H}]$} & $\mathrm{Mem}^{\mathrm{b}}$ \\
\hline DES J220255.41-462538.9 & 330.73088 & -46.42747 & 19.18 & 19.24 & 58363.6 & 5.9 & $-104.4 \pm 3.3$ & $\ldots$ & $\ldots$ & 1 \\
\hline DES J220304.94-462841.7 & 330.77059 & -46.47824 & 20.59 & 20.09 & 57282.6 & 11.8 & $-142.7 \pm 4.1$ & $5.98 \pm 0.61$ & $\cdots$ & 0 \\
\hline DES J220308.10-462259.2 & 330.78377 & -46.38312 & 20.15 & 19.25 & 58363.6 & 8.6 & $107.3 \pm 2.7$ & $\cdots$ & $\cdots$ & 0 \\
\hline DES J220309.37-462634.2 & 330.78903 & -46.44283 & 18.44 & 17.57 & 58363.6 & 34.5 & $23.4 \pm 1.2$ & $5.65 \pm 0.38$ & $\cdots$ & 0 \\
\hline \multirow[t]{2}{*}{ DES J220309.47-463255.6 } & 330.78944 & -46.54878 & 18.69 & 18.08 & 57282.6 & 45.7 & $40.4 \pm 1.2$ & $5.75 \pm 0.36$ & $\cdots$ & 0 \\
\hline & 330.78944 & -46.54878 & 18.69 & 18.08 & 57634.4 & 18.4 & $41.8 \pm 1.5$ & $5.76 \pm 0.49$ & $\cdots$ & 0 \\
\hline DES J220310.98-462848.8 & 330.79573 & -46.48023 & 18.78 & 18.43 & 58363.6 & 16.6 & $42.2 \pm 1.4$ & $3.95 \pm 0.45$ & $\cdots$ & 0 \\
\hline DES J220311.39-462555.3 & 330.79746 & -46.43203 & 19.48 & 19.12 & 58363.6 & 8.3 & $18.3 \pm 1.7$ & $2.56 \pm 0.55$ & $\cdots$ & 0 \\
\hline DES J220311.75-462652.7 & 330.79897 & -46.44796 & 21.15 & 20.64 & 57980.9 & 5.6 & $69.2 \pm 3.2$ & $4.01 \pm 1.81$ & $\cdots$ & 0 \\
\hline \multirow[t]{2}{*}{ DES J220312.90-463312.1 } & 330.80374 & -46.55336 & 19.61 & 18.95 & 57282.6 & 26.1 & $13.9 \pm 1.4$ & $5.75 \pm 0.41$ & $\cdots$ & 0 \\
\hline & 330.80374 & -46.55336 & 19.61 & 18.95 & 57634.4 & 8.4 & $16.0 \pm 2.9$ & $\cdots$ & $\cdots$ & 0 \\
\hline
\end{tabular}

Notes.

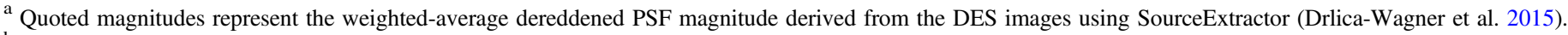

${ }^{\mathrm{b}} \mathrm{A}$ value of 1 indicates that the star is a member of the relevant satellite, while 0 indicates a nonmember.

(This table is available in its entirety in machine-readable form.)

properties match the mean properties of the satellites so closely, all of our conclusions would be unaffected if they were included as members. A second Tuc IV candidate (DES J000244.67 -604819.1) that is on the RGB in the CMD and located near the center of the system is a $\sim 2 \sigma$ outlier in both velocity and proper motion, as well as having a relatively high $\mathrm{CaT}$ metallicity of $[\mathrm{Fe} / \mathrm{H}]=-1.43$, so we judge it a nonmember. If it were a member of Tuc IV, it would increase the inferred velocity dispersion by less than $1 \sigma$. Our spectroscopic data sets include 21 members of Gru II, 11 members of Tuc IV, and 3 members of Tuc V.

All Magellan/IMACS velocity and metallicity measurements, as well as membership determinations, are listed in Table 3.

\subsection{Binarity}

For member stars that were observed on at least two separate observing runs, we check for velocity variations between measurements using a $\chi^{2}$ test. In most cases, the data are consistent with the null hypothesis that the velocity of the star is constant with time. We obtained multiple measurements of 15 of the 21 members of Gru II. Two out of those fifteen stars have probabilities of a constant velocity low enough $(p \ll 0.01)$ for that hypothesis to be confidently rejected: the red giant DES J220352.01-462446.5 $\left(\Delta V=10.0 \pm 1.7 \mathrm{~km} \mathrm{~s}^{-1}\right)$ and the blue horizontal branch (BHB) star DES J220433.75 $-462639.8\left(\Delta V=14.5 \pm 3.3 \mathrm{~km} \mathrm{~s}^{-1}\right) .{ }^{46}$ Several other stars exhibit weaker evidence for variability, with $0.01 \leqslant p \leqslant 0.10$.

For 8 of the 11 members of Tuc IV, we obtained multiple spectra separated by at least $\sim 1$ month. Two of these stars exhibit clear velocity changes during the course of our observations

\footnotetext{
${ }^{46}$ For the latter star, this velocity difference is measured between two spectra obtained a few hours apart during the same observing run. It is therefore not clear whether to interpret the velocity measurements as evidence of a shortperiod binary system or evidence that one of the measurements is erroneous. An additional velocity measurement for this star obtained $\sim 1$ yr later agrees with the first measurement.
} 
(DES J000228.19-604814.3, $\Delta V=15.7 \pm 3.1 \mathrm{~km} \mathrm{~s}^{-1}$; DES J000119.59-604439.2, $\Delta V=13.0 \pm 2.8 \mathrm{~km} \mathrm{~s}^{-1}$ ), and a third star with $p=0.03$ (DES J000303.55-605025.3) may be variable as well. The first of these is a BHB star likely with an eccentric orbit, as we observe slow velocity changes over several months in 2015 and a change of $\sim 10 \mathrm{~km} \mathrm{~s}^{-1}$ over less than four weeks in 2018. The other five members show no significant evidence for radial velocity variability. Finding two clear binaries out of seven stars in Tuc IV is a larger percentage of radial velocity variables than is seen in other dwarf galaxies, although of course the binomial uncertainty is large with so few stars. Nevertheless, this result could suggest that the binary fraction of Tuc IV is very high, consistent with recent determinations of the close binary fraction as a function of metallicity in the Milky Way (Moe et al. 2019) and with estimates of the binary population in several satellite galaxies (Geha et al. 2013; Spencer et al. 2018; Minor et al. 2019). We observed two of the three Tuc $\mathrm{V}$ members with a time baseline of $\sim 1 \mathrm{yr}$, with no evidence for velocity changes for either star.

\section{Results}

\subsection{Structural Properties}

The luminosity, size, and other photometric parameters of our three target satellites were originally determined from the DES year 2 quick release catalog (Drlica-Wagner et al. 2015) and have not been updated with deeper data by other authors. ${ }^{47}$ Because Tuc IV and Tuc V are close to the detection limits of DES in surface brightness and luminosity, respectively, we repeat the Drlica-Wagner et al. (2015) analysis of all three systems with the deeper and more uniform DES Y3A2 catalog (DES Collaboration et al. 2018). The updated parameters for each object are listed in Tables 4-6. Relative to the DrlicaWagner et al. results, the luminosity of all three satellites is lower by $\gtrsim 30 \%$, although the error bars of the Y2 and Y3 luminosities overlap. For Tuc IV, we find a somewhat smaller half-light radius of 9!3. For Tuc $\mathrm{V}$, we find a larger half-light radius of 2!'1. The larger size of Tuc V may explain why Conn et al. (2018) were unable to identify a clear overdensity in the small Gemini/GMOS field of view they employed.

\subsection{Stellar Kinematics}

Given the member samples identified in Section 3.3, we use the maximum likelihood approach defined in Simon et al. (2017) and Li et al. (2017) to determine the mean velocity and velocity dispersion of each of the three satellites. In cases where we have multiple measurements of a star separated by more than $\sim 1$ month, we average all of the measurements together before computing the global properties.

For Gru II, with the two binaries removed, we measure a systemic velocity of $v_{\text {hel }}=-110.0 \pm 0.5 \mathrm{~km} \mathrm{~s}^{-1}$ and a velocity dispersion of $\sigma=1.2 \pm 0.6 \mathrm{~km} \mathrm{~s}^{-1}$ ( $1 \sigma$ uncertainties). However, at $\sim 2 \sigma$, the dispersion is consistent with zero, so our data do not clearly resolve the internal kinematics of Gru II. We place a 90\% (95.5\%) confidence upper limit on the velocity dispersion of $1.9 \mathrm{~km} \mathrm{~s}^{-1}\left(2.0 \mathrm{~km} \mathrm{~s}^{-1}\right)$. The posterior probability distributions for the velocity and velocity dispersion are estimated using an affine-invariant Markov Chain Monte Carlo ensemble sampler (Foreman-Mackey et al. 2013) and are

\footnotetext{
$\overline{47}$ Conn et al. (2018) did obtain deep Gemini imaging of Tuc V, but were not able to characterize it (see below and Section 5).
}

Table 4

Summary of Properties of Grus II

\begin{tabular}{|c|c|c|}
\hline Row & Quantity & Value \\
\hline (1) & R.A. (J2000) & $331.025_{-0.008}^{+0.009}$ \\
\hline (2) & Decl. (J2000) & $-46.442 \pm 0.006$ \\
\hline (3) & Distance $(\mathrm{kpc})^{\mathrm{a}}$ & $55 \pm 2$ \\
\hline (4) & $r_{1 / 2}(\operatorname{arcmin})^{\mathrm{b}}$ & $5.9 \pm 0.5$ \\
\hline (5) & Ellipticity & $<0.21$ \\
\hline (6) & Position angle (degrees) & $\cdots$ \\
\hline (7) & $M_{V, 0}$ & $-3.5 \pm 0.3$ \\
\hline (8) & $L_{V, 0}\left(L_{\odot}\right)$ & $2100_{-500}^{+700}$ \\
\hline (9) & $r_{1 / 2}(\mathrm{pc})$ & $94 \pm 9$ \\
\hline (10) & $N_{\text {spectroscopic members }}$ & 21 \\
\hline (11) & $V_{\text {hel }}\left(\mathrm{km} \mathrm{s}^{-1}\right)$ & $-110.0 \pm 0.5$ \\
\hline (12) & $V_{\mathrm{GSR}}\left(\mathrm{km} \mathrm{s}^{-1}\right)$ & $-132.0 \pm 0.5$ \\
\hline (13) & $\sigma\left(\mathrm{km} \mathrm{s}^{-1}\right)^{\mathrm{c}}$ & $<2.0$ \\
\hline (14) & $\operatorname{Mass}\left(M_{\odot}\right)^{\mathrm{c}}$ & $<3.5 \times 10^{5}$ \\
\hline (15) & $M / L_{V}\left(M_{\odot} / L_{\odot}\right)^{\mathrm{c}}$ & $<330$ \\
\hline (16) & Mean $[\mathrm{Fe} / \mathrm{H}]$ & $-2.51 \pm 0.11$ \\
\hline (17) & Metallicity dispersion $(\mathrm{dex})^{\mathrm{c}}$ & $<0.45$ \\
\hline (18) & $\mu_{\alpha} \cos \delta\left(\operatorname{mas}_{\mathrm{yr}^{-1}}\right)$ & $0.45 \pm 0.08$ \\
\hline (19) & $\mu_{\delta}\left(\operatorname{mas~yr}^{-1}\right)$ & $-1.46 \pm 0.09$ \\
\hline (20) & Orbital periright $(\mathrm{kpc})$ & $25_{-7}^{+6}$ \\
\hline (21) & Orbital aporight $(\mathrm{kpc})$ & $66_{-5}^{+7}$ \\
\hline (22) & $\log _{10} J(0.2)\left(\mathrm{GeV}^{2} \mathrm{~cm}^{-5}\right)^{\mathrm{c}}$ & $<16.5$ \\
\hline (23) & $\log _{10} J(0.5)\left(\mathrm{GeV}^{2} \mathrm{~cm}^{-5}\right)^{\mathrm{c}}$ & $<16.7$ \\
\hline
\end{tabular}

Notes.

${ }^{a}$ We adopt the RR Lyrae distance to Grus II from Martínez-Vázquez et al. (2019). The RR Lyrae-based measurement is consistent with the isochrone distance but has a smaller uncertainty.

${ }^{\mathrm{b}}$ The radius listed here is the semimajor axis of the half-light ellipse (referred to as $a_{\mathrm{h}}$ in Drlica-Wagner et al. 2015).

${ }^{\mathrm{c}}$ Upper limits listed here are at $95.5 \%$ confidence. See the text for values at other confidence levels.

displayed on the left side of Figure 6. Using the relation given by Wolf et al. (2010) and the upper limit on the velocity dispersion of Gru II, the corresponding (90\% confidence) upper limit on the mass enclosed within its half-light radius is $3.2 \times 10^{5} M_{\odot}\left(M / L<300 M_{\odot} / L_{\odot}\right.$ within the half-light radius).

After excluding binary stars, ${ }^{48}$ we measure a systemic velocity of $v_{\text {hel }}=15.9_{-1.7}^{+1.8} \mathrm{~km} \mathrm{~s}^{-1}$ and a velocity dispersion of $\sigma=4.3_{-1.0}^{+1.7} \mathrm{~km} \mathrm{~s}^{-1}$ for Tuc IV. Despite the small sample of member stars, the dispersion of Tuc IV is significantly resolved; at $2 \sigma$, the velocity dispersion is larger than $2.7 \mathrm{~km} \mathrm{~s}^{-1}$. The Monte Carlo posteriors are displayed on the right side of Figure 6. Because the velocity of Tuc IV coincides with a substantial fraction of the Milky Way foreground stars, one might worry that its velocity dispersion is being inflated by contamination of the member sample. However, because the stars classified as members are all either BHB stars, where contamination should be negligible (see Figure 2(a)), or are RGB stars with both metallicities and proper motions consistent with membership, such contaminants are unlikely. The nonzero velocity dispersion in Tuc IV is based primarily on the velocities of three stars (DES J000233.86-605439.4,

\footnotetext{
${ }^{48}$ Including the two binaries actually slightly decreases the measured dispersion, at least in part because one of them has enough velocity measurements that their average is a reasonable estimate of the center-ofmass velocity of the binary.
} 
Table 5

Summary of Properties of Tucana IV

\begin{tabular}{|c|c|c|}
\hline Row & Quantity & Value \\
\hline (1) & R.A. (J2000) & $0.717_{-0.021}^{+0.014}$ \\
\hline (2) & Decl. (J2000) & $-60.830_{-0.011}^{+0.010}$ \\
\hline (3) & Distance (kpc) & $47 \pm 4$ \\
\hline (4) & $r_{1 / 2}(\operatorname{arcmin})^{\mathrm{a}}$ & $9.3_{-0.9}^{+1.4}$ \\
\hline (5) & Ellipticity & $0.39_{-0.10}^{+0.07}$ \\
\hline (6) & Position angle (degrees) & $27_{-8}^{+9}$ \\
\hline (7) & $M_{V, 0}$ & $-3.0_{-0.4}^{+0.3}$ \\
\hline (8) & $L_{V, 0}\left(L_{\odot}\right)$ & $1400_{-300}^{+600}$ \\
\hline (9) & $r_{1 / 2}(\mathrm{pc})$ & $127_{-16}^{+22}$ \\
\hline (10) & $N_{\text {spectroscopic members }}$ & 11 \\
\hline (11) & $V_{\text {hel }}\left(\mathrm{km} \mathrm{s}^{-1}\right)$ & $15.9_{-1.7}^{+1.8}$ \\
\hline (12) & $V_{\mathrm{GSR}}\left(\mathrm{km} \mathrm{s}^{-1}\right)$ & $-82.9_{-1.7}^{+1.8}$ \\
\hline (13) & $\sigma\left(\mathrm{km} \mathrm{s}^{-1}\right)$ & $4.3_{-1.0}^{+1.7}$ \\
\hline (14) & Mass $\left(M_{\odot}\right)$ & $2.2_{-1.1}^{+1.8} \times 10^{6}$ \\
\hline (15) & $M / L_{V}\left(M_{\odot} / L_{\odot}\right)$ & $3100_{-1600}^{+2900}$ \\
\hline (16) & Mean $[\mathrm{Fe} / \mathrm{H}]$ & $-2.49_{-0.16}^{+0.15}$ \\
\hline (17) & Metallicity dispersion $(\mathrm{dex})^{\mathrm{b}}$ & $<0.64$ \\
\hline (18) & $\mu_{\alpha} \cos \delta\left(\operatorname{mas} \mathrm{yr}^{-1}\right)$ & $0.51 \pm 0.14$ \\
\hline (19) & $\mu_{\delta}\left(\operatorname{mas} \mathrm{yr}^{-1}\right)$ & $-1.64 \pm 0.13$ \\
\hline (20) & Orbital pericenter $(\mathrm{kpc})$ & $25_{-8}^{+11}$ \\
\hline (21) & Orbital apocenter $(\mathrm{kpc})$ & $52_{-6}^{+12}$ \\
\hline (22) & $\log _{10} J(0.2)\left(\mathrm{GeV}^{2} \mathrm{~cm}^{-5}\right)$ & $18.2_{-0.5}^{+0.6}$ \\
\hline (23) & $\log _{10} J(0.5)\left(\mathrm{GeV}^{2} \mathrm{~cm}^{-5}\right)$ & $18.4_{-0.5}^{+0.6}$ \\
\hline
\end{tabular}

Notes.

${ }^{\mathrm{a}}$ The radius listed here is the semimajor axis of the half-light ellipse (referred to as $a_{\mathrm{h}}$ in Drlica-Wagner et al. 2015).

${ }^{\mathrm{b}}$ Upper limits listed here are at $95.5 \%$ confidence. See the text for values at other confidence levels.

DES J000251.92-604820.8, and DES J000344.06 -604804.7 ), the first two of which have velocities $\sim 1.5 \sigma$ below the mean velocity of the system, and the third $\sim 1.5 \sigma$ above the mean velocity. If (some of) these stars are binaries, the actual intrinsic dispersion of Tuc IV could be smaller. However, we have multiple velocity measurements of each star spanning from 3 months (DES J000344.06-604804.7) to $3 \mathrm{yr}$ for the other two, with no evidence of radial velocity variations, so we can rule out that they have binary companions with periods shorter than a few years.

The mass within the half-light radius of Tuc IV according to the Wolf et al. (2010) estimator is $2.2_{-1.1}^{+1.8} \times 10^{6} M_{\odot}$. However, we note that the uncertainty on the mass is non-Gaussian, so the $2 \sigma$ uncertainty is not twice as large as the $1 \sigma$ uncertainty. Even using the $2 \sigma$ lower limit on the velocity dispersion, the half-light mass of Tuc IV is large $\left(8.6 \times 10^{5} M_{\odot}\right)$. For comparison, the total luminosity of Tuc IV is $1400 L_{\odot}$, indicating a highly dark-matter-dominated system.

From the three members of Tuc V, we determine a systemic velocity of $v_{\text {hel }}=-36.2_{-2.2}^{+2.5} \mathrm{~km} \mathrm{~s}^{-1}$. Given the small sample and the similar velocities of the three stars, we cannot place any significant constraints on the velocity dispersion of the system (the $2 \sigma$ upper limit is $7.4 \mathrm{~km} \mathrm{~s}^{-1}$ ). Substantially deeper spectroscopy will be needed to study the internal kinematics of Tuc V. The posterior probability distributions are displayed in Figure 7. The mass of Tuc V is not usefully constrained by the data.
Table 6

Summary of Properties of Tucana V

\begin{tabular}{|c|c|c|}
\hline Row & Quantity & Value \\
\hline (1) & R.A. (J2000) & $354.347 \pm 0.008$ \\
\hline (2) & Decl. (J2000) & $-63.266_{-0.004}^{+0.006}$ \\
\hline (3) & Distance $(\mathrm{kpc})$ & $55_{-8}^{+3}$ \\
\hline (4) & $r_{1 / 2}(\operatorname{arcmin})^{\mathrm{a}}$ & $2.1_{-0.4}^{+0.6}$ \\
\hline (5) & Ellipticity & $0.51_{-0.18}^{+0.09}$ \\
\hline (6) & Position angle (degrees) & $29 \pm 11$ \\
\hline (7) & $M_{V, 0}$ & $-1.1_{-0.6}^{+0.5}$ \\
\hline (8) & $L_{V, 0}\left(L_{\odot}\right)$ & $240_{-90}^{+170}$ \\
\hline (9) & $r_{1 / 2}(\mathrm{pc})$ & $34_{-8}^{+11}$ \\
\hline (10) & $N_{\text {spectroscopic members }}$ & 3 \\
\hline (11) & $V_{\text {hel }}\left(\mathrm{km} \mathrm{s}^{-1}\right)$ & $-36.2_{-2.2}^{+2.5}$ \\
\hline (12) & $V_{\mathrm{GSR}}\left(\mathrm{km} \mathrm{s}^{-1}\right)$ & $-136.6_{-2.2}^{+2.5}$ \\
\hline (13) & $\sigma\left(\mathrm{km} \mathrm{s}^{-1}\right)^{\mathrm{b}}$ & $<7.4$ \\
\hline (14) & Mass $\left(M_{\odot}\right)^{\mathrm{b}}$ & $<1.7 \times 10^{6}$ \\
\hline (15) & $M / L_{V}\left(M_{\odot} / L_{\odot}\right)^{\mathrm{b}}$ & $<14000$ \\
\hline (16) & Mean $[\mathrm{Fe} / \mathrm{H}]$ & $-2.17 \pm 0.23$ \\
\hline (17) & $\mu_{\alpha} \cos \delta\left(\operatorname{mas} \mathrm{yr}^{-1}\right)$ & $-0.62 \pm 0.31$ \\
\hline (18) & $\mu_{\delta}\left(\operatorname{mas} \mathrm{yr}^{-1}\right)$ & $-0.88 \pm 0.35$ \\
\hline (19) & Orbital pericenter $(\mathrm{kpc})$ & $36_{-15}^{+13}$ \\
\hline (20) & Orbital apocenter (kpc) & $131_{-60}^{+470}$ \\
\hline (21) & $\log _{10} J(0.2)\left(\mathrm{GeV}^{2} \mathrm{~cm}^{-5}\right)^{\mathrm{b}}$ & $<19.4$ \\
\hline (22) & $\log _{10} J\left(0^{\circ} .5\right)\left(\mathrm{GeV}^{2} \mathrm{~cm}^{-5}\right)^{\mathrm{b}}$ & $<19.6$ \\
\hline
\end{tabular}

Notes.

${ }^{\mathrm{a}}$ The radius listed here is the semimajor axis of the half-light ellipse (referred to as $a_{\mathrm{h}}$ in Drlica-Wagner et al. 2015).

${ }^{\mathrm{b}}$ Upper limits listed here are at $95.5 \%$ confidence.

\subsection{Metallicities and Metallicity Spreads}

We use the same maximum likelihood method to determine the metallicity distribution of each satellite. The RGB stars in Gru II have a mean metallicity of $[\mathrm{Fe} / \mathrm{H}]=-2.51 \pm 0.11$, with a dispersion of $\sigma_{[\mathrm{Fe} / \mathrm{H}]}=0.21_{-0.13}^{+0.15}$ dex. The $2 \sigma$ upper limit on the metallicity spread is 0.45 dex. The mean metallicity of Tuc IV is nearly identical, at $[\mathrm{Fe} / \mathrm{H}]=-2.49_{-0.16}^{+0.15}$. The metallicity dispersion for the eight RGB stars in Tuc IV is $\sigma_{[\mathrm{Fe} / \mathrm{H}]}=0.18_{-0.18}^{+0.20} \mathrm{dex}$, with a $2 \sigma$ upper limit of 0.64 dex. The measurements for Tuc $\mathrm{V}$ are also similar, with $[\mathrm{Fe} / \mathrm{H}]=-2.17 \pm 0.23$ and a metallicity dispersion that cannot be significantly constrained with only two metallicity measurements (the signal-to-noise ratio for the third Tuc $\mathrm{V}$ member star is too low for a reliable determination of the CaT EW).

\subsection{Proper Motions and Orbits}

Given a set of spectroscopic members in each object, we can use the astrometry provided by the second data release (Gaia Collaboration et al. 2018) from the Gaia mission (Gaia Collaboration et al. 2016) to measure mean proper motions, as in Simon (2018). Massari \& Helmi (2018) and Pace \& Li (2019) already estimated the proper motions of Gru II and Tuc IV using photometrically selected member samples, but our spectroscopic samples offer a cleaner and more robust determination. Using only spectroscopic members, we measure proper motions of $\mu_{\alpha} \cos \delta=0.45 \pm 0.08 \operatorname{mas~yr}^{-1}, \quad \mu_{\delta}=-1.46 \pm 0.09$

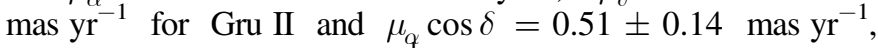

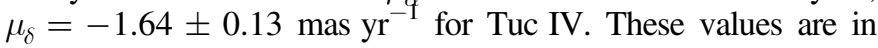



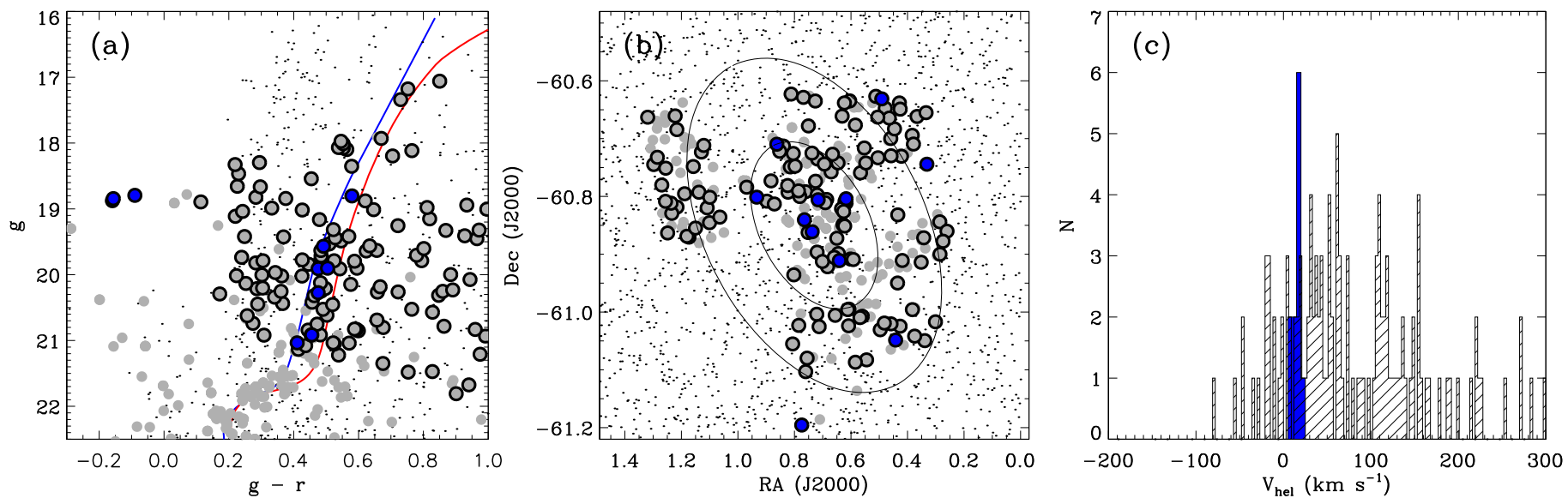

Figure 2. (a) DES CMD of Tucana IV. Stars within $17^{\prime}$ of the center of Tuc IV are plotted as small black dots, and stars selected for spectroscopy (as described in Section 2.2) are plotted as filled gray circles. Points surrounded by black outlines represent the stars for which we obtained successful velocity measurements, and those we identify as Tuc IV members are filled in with blue. The M92 sequence and PARSEC isochrone used to define the RGB are displayed as blue and red curves, respectively. (b) Spatial distribution of the observed stars. Symbols are as in panel (a). The black ellipses represent one and two times the half-light radius of Tuc IV. (c) Radial velocity distribution of observed stars. Tuc IV members are highlighted in blue, and the hatched histogram indicates stars that are not members of Tuc IV.

excellent agreement with the determinations of Pace \& Li (2019), as well as with the Massari \& Helmi (2018) measurements in the decl. direction, but deviate by $\sim 1.5 \sigma$ from Massari \& Helmi (2018) in the R.A. direction. We find that up to $\sim 1 / 4$ of the Gru II stars and $\sim 1 / 3$ of the Tuc IV stars assumed to be members by Massari \& Helmi (2018) are not spectroscopic members, which may explain this discrepancy. For Tuc V, which has no previous proper motion measurement, we derive $\mu_{\alpha} \cos \delta=$

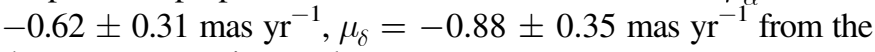
three spectroscopic members.

\subsubsection{Orbits in the Milky Way Potential}

In combination with the radial velocities measured here (Section 4.2) and the distances listed in Tables 4-6, these proper motions determine the orbits of the three satellites around the Milky Way. As a starting point, we adopt the modified MWPotential2014 (where the original MWPotential2014 is taken from Bovy 2015) gravitational potential defined by Carlin \& Sand (2018), with a total mass for the Milky Way of $1.6 \times 10^{12} M_{\odot}$ (e.g., Watkins et al. 2019). We integrate orbits with galpy (Bovy 2015) as described in Simon (2018). The orbit of Gru II is tightly bound and well determined, with a pericenter of $25_{-7}^{+6} \mathrm{kpc}$ (where the confidence interval is determined from 1000 Monte Carlo iterations, drawing input distances, radial velocities, and proper motions from distributions set by the measured values and their uncertainties), an apocenter of $66_{-5}^{+7} \mathrm{kpc}$, an eccentricity of $0.45_{-0.05}^{+0.08}$, and an orbital period of $\sim 0.9$ Gyr. Gru II has likely completed many orbits around the Galaxy, and it is currently approaching pericenter, which it will reach in $\sim 200 \mathrm{Myr}$. The situation for Tuc IV is similar, with a best-fit orbital pericenter of $25_{-8}^{+11} \mathrm{kpc}$, an apocenter of $52_{-6}^{+12} \mathrm{kpc}$, an eccentricity of $0.36_{-0.06}^{+0.13}$, and an orbital period of $\sim 0.7$ Gyr. Like Gru II, Tuc IV is approaching the pericenter of its orbit and will reach that point in $\sim 200 \mathrm{Myr}$. These two satellites lessen the previously observed tendency of known ultra-faint dwarfs to be discovered near their pericenters (Fritz et al. 2018; Simon 2018).

Tuc V has a somewhat larger orbital pericenter than the other two systems, $35_{-13}^{+10} \mathrm{kpc}$, and its best-fit apocenter reaches well out into the halo of the Milky Way $\left(d_{\text {apo }}=126_{-49}^{+243} \mathrm{kpc}\right)$. The corresponding orbital eccentricity is $0.59_{-0.07}^{+0.21}$. Tuc V has a median orbital period of $1.3 \mathrm{Gyr}$, but in $\sim 20 \%$ of the Monte Carlo iterations, Tuc V is approaching the Milky Way for the first time. Its next (or first) pericenter will occur in 145 Myr.

In order to test the robustness of these orbital results, we also compute the orbits of the three satellites in the McMillan (2017) Milky Way potential using GALPOT (Dehnen \& Binney 1998). We find that most of the orbital parameters are within the $1 \sigma$ ranges given in Tables 4-6.

\subsubsection{A Collision with the LMC}

To assess whether any of the three satellites could be associated with the Magellanic Clouds, we integrate the derived orbits and that of the Large Magellanic Cloud (LMC) backward in the Milky Way potential, as shown in Figure 8. For the LMC, we adopt the proper motion from Kallivayalil et al. (2013), the radial velocity from van der Marel et al. (2002), and we assume a distance of $49.97 \pm 1.13 \mathrm{kpc}$ from Pietrzyński et al. (2013). Gru II and Tuc V follow very different paths from the LMC and are unlikely to be associated with it (see Section 5.3 for a more detailed discussion). We find that Tuc IV, on the other hand, passed $5.8 \mathrm{kpc}$ from the center of the LMC with a relative velocity of $\sim 200 \mathrm{~km} \mathrm{~s}^{-1} \sim 130 \mathrm{Myr}$ ago. This close encounter may have both deflected Tuc IV from its previous trajectory and disturbed its internal kinematics, and renders our simple calculation ignoring the gravitational potential of the LMC incomplete. For comparison, although Tuc $\mathrm{V}$ also had a relatively close approach to the LMC of $16.5 \mathrm{kpc} \sim 50 \mathrm{Myr}$ ago, the relative velocity between the two was $\sim 460 \mathrm{~km} \mathrm{~s}^{-1}$, suggesting that it is not a Magellanic satellite. For the rest of this section, we will only focus on Tuc IV because Gru II and Tuc V are not strongly affected by the LMC.

In order to include the effect of the Magellanic Clouds, we repeat the orbit calculations following a similar approach to those described by Erkal et al. $(2018,2019)$ and Erkal \& Belokurov (2019). Motivated by the measurement of the LMC mass in Erkal et al. (2019), we model the LMC as a Hernquist profile (Hernquist 1990) with a mass of $1.5 \times 10^{11} M_{\odot}$ and a 

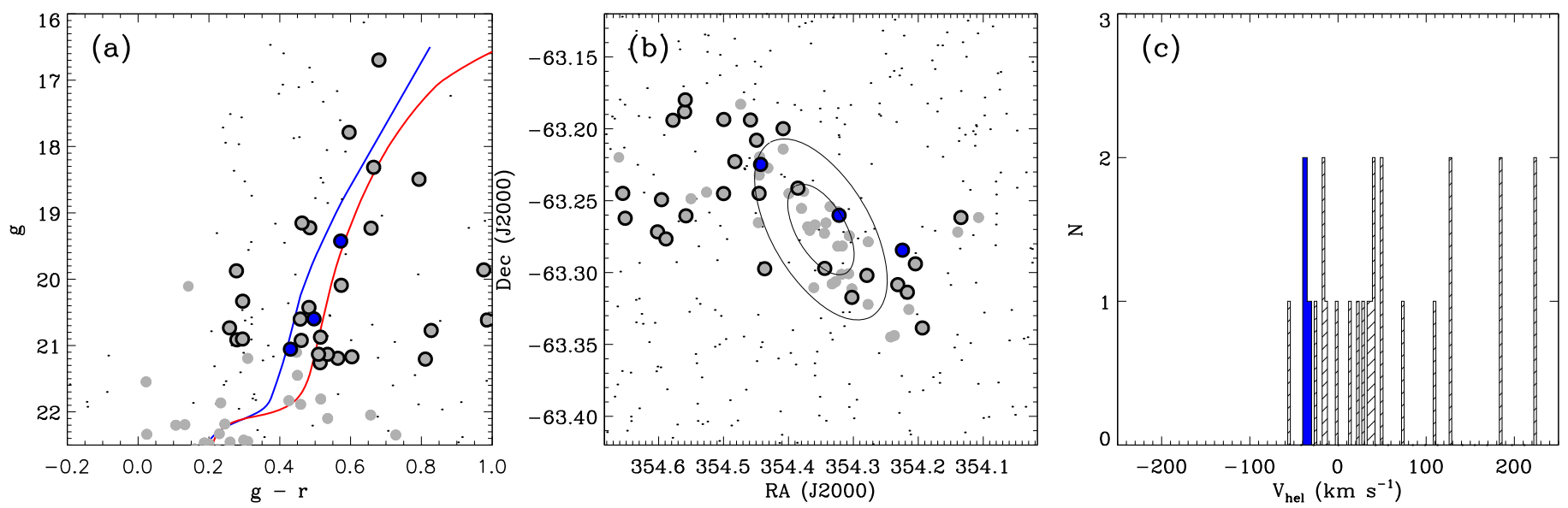

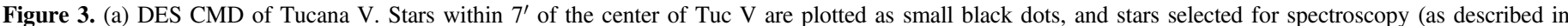

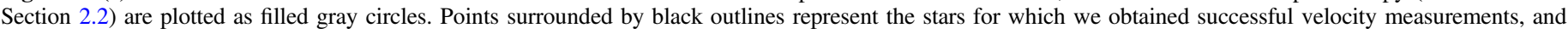

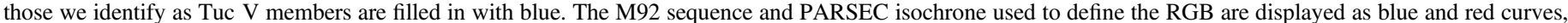

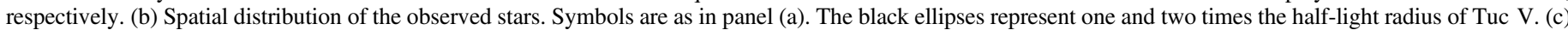

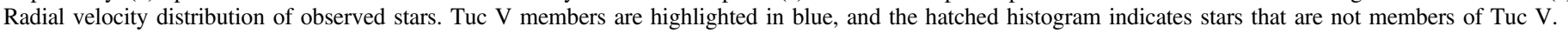
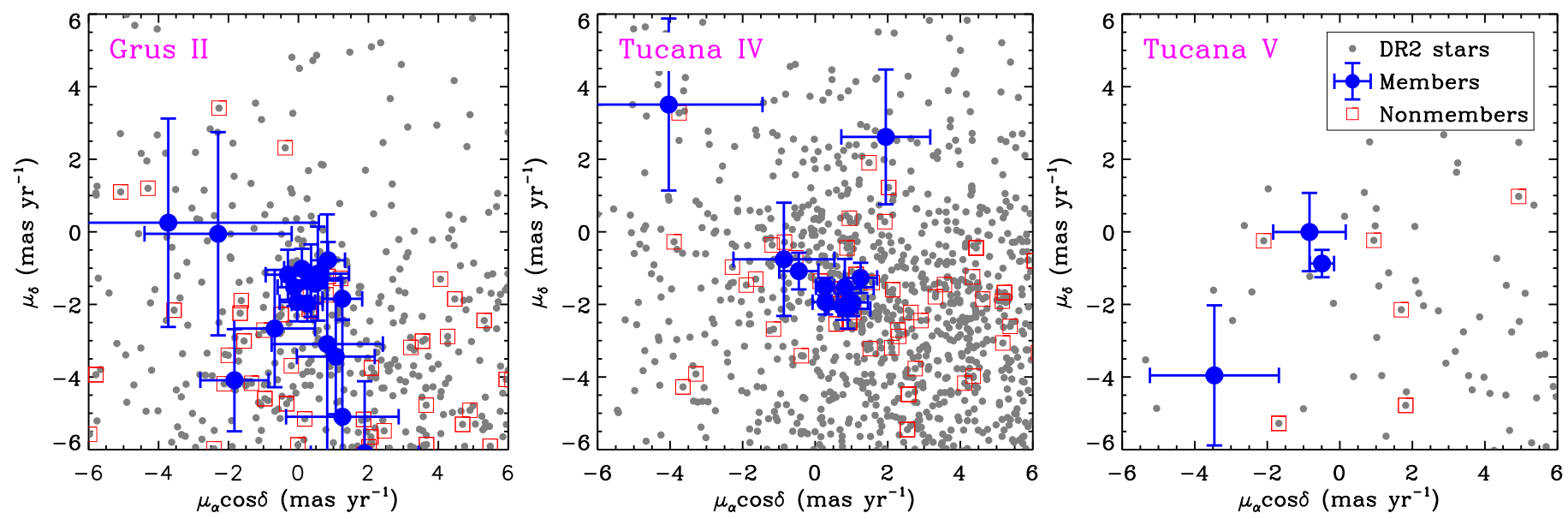

Figure 4. Proper motion diagrams for Gru II (left), Tuc IV (middle), and Tuc V (right). The identified members of each satellite are plotted as filled blue circles, while spectroscopic nonmembers are shown as open red squares. The small gray points represent other stars in the Gaia DR 2 catalog within $3 r_{1 / 2}$ of each system for which we did not obtain spectra. The tight clustering of the member stars in proper motion is evident for Gru II and Tuc IV. Two faint members of Tuc IV and one of Tuc V are near the magnitude limit of the Gaia catalog and appear as outliers here, but given the large astrometric uncertainties, their proper motions are within $\sim 2 \sigma$ of the mean value.

scale radius of $17.13 \mathrm{kpc}$. We use the same potential for the Milky Way as in Section 4.4.1 and account for the reflex motion of the Milky Way in response to the LMC as in Erkal et al. (2019). We find that Tuc IV collided with the LMC with a closest approach of $4.1_{-2.2}^{+3.2} \mathrm{kpc}$ and a relative velocity of $281_{-39}^{+26} \mathrm{~km} \mathrm{~s}^{-1}$ at a time of $119_{-18}^{+26} \mathrm{Myr}$ ago.

As in Erkal \& Belokurov (2019), this rewinding procedure can be used to determine whether Tuc IV was originally an LMC satellite by computing the likelihood that Tuc IV was bound to the LMC prior to their encounter. The resulting low probability of 3.8\% suggests that it is not a Magellanic satellite. However, given the close passage of Tuc IV with respect to the LMC, it is natural to include the Small Magellanic Cloud (SMC) as well. For the SMC, we use the proper motion from Kallivayalil et al. (2013), the radial velocity from Harris \& Zaritsky (2006), and the distance from Graczyk et al. (2014). If we treat the SMC as a Hernquist profile with a mass of $10^{10} M_{\odot}$ and a scale radius of $1 \mathrm{kpc}$, the probability that Tuc IV was originally bound to the
LMC rises to $18.1 \%$. While this probability is still modest compared to the satellites classified as having a Magellanic origin by Erkal \& Belokurov (2019; $\gtrsim 50 \%$ ), we have rerun the analysis of Erkal and Belokurov including the SMC, and we find that Tuc IV is the only satellite for which including the SMC has an appreciable effect. The closest approach between Tuc IV and the SMC is $6.6_{-3.7}^{+5.3} \mathrm{kpc}$, which is comparable to the closest approach with the LMC. This result suggests that Tuc IV may have actually undergone a three-body interaction with both Magellanic Clouds, which can be tested once improved proper motions are available.

Given the close passage of Tuc IV with respect to the LMC, we note that although the Hernquist profile we have assumed for the LMC matches the observed rotation curve from van der Marel \& Kallivayalil (2014) at $8.7 \mathrm{kpc}$, the modeled rotation curve falls below the observed one at smaller radii. Thus, we may be underpredicting the effect of the LMC on Tuc IV. In order to assess the importance of this discrepancy, we also 

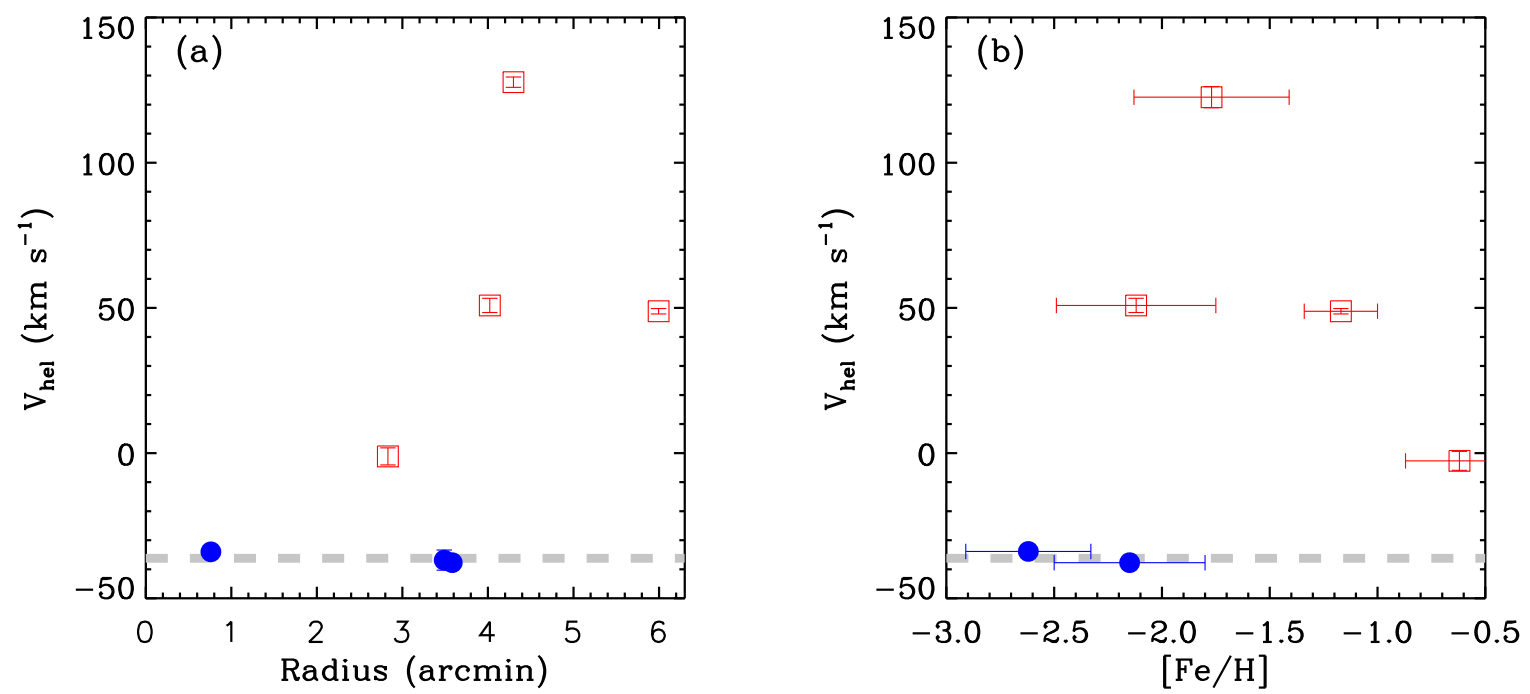

Figure 5. (Left) Velocity as a function of distance away from the center of Tuc V for stars in the RGB selection region shown in Figure 3(a). The identified Tuc V members are plotted as filled blue circles, while the spectroscopic nonmembers are shown as open red squares. The systemic velocity of Tuc $\mathrm{V}$ is displayed as a dashed gray line. (Right) Velocity as a function of metallicity for stars in the RGB selection region (between the two isochrones and brighter than $g \approx 21.1$ ) shown in Figure 3(a). Symbols are as in the left panel. The spectrum of the faintest Tuc V member does not have a high enough S/N for an accurate measurement of the CaT EW, so only the two brighter members are plotted in this panel. Note that because CaT metallicities depend on the assumed distance, the metallicities for the nonTuc V stars are likely underestimated (presuming that these stars are in the foreground). The Tuc V members share a common velocity and are closer to the center and more metal-poor than the nonmembers.
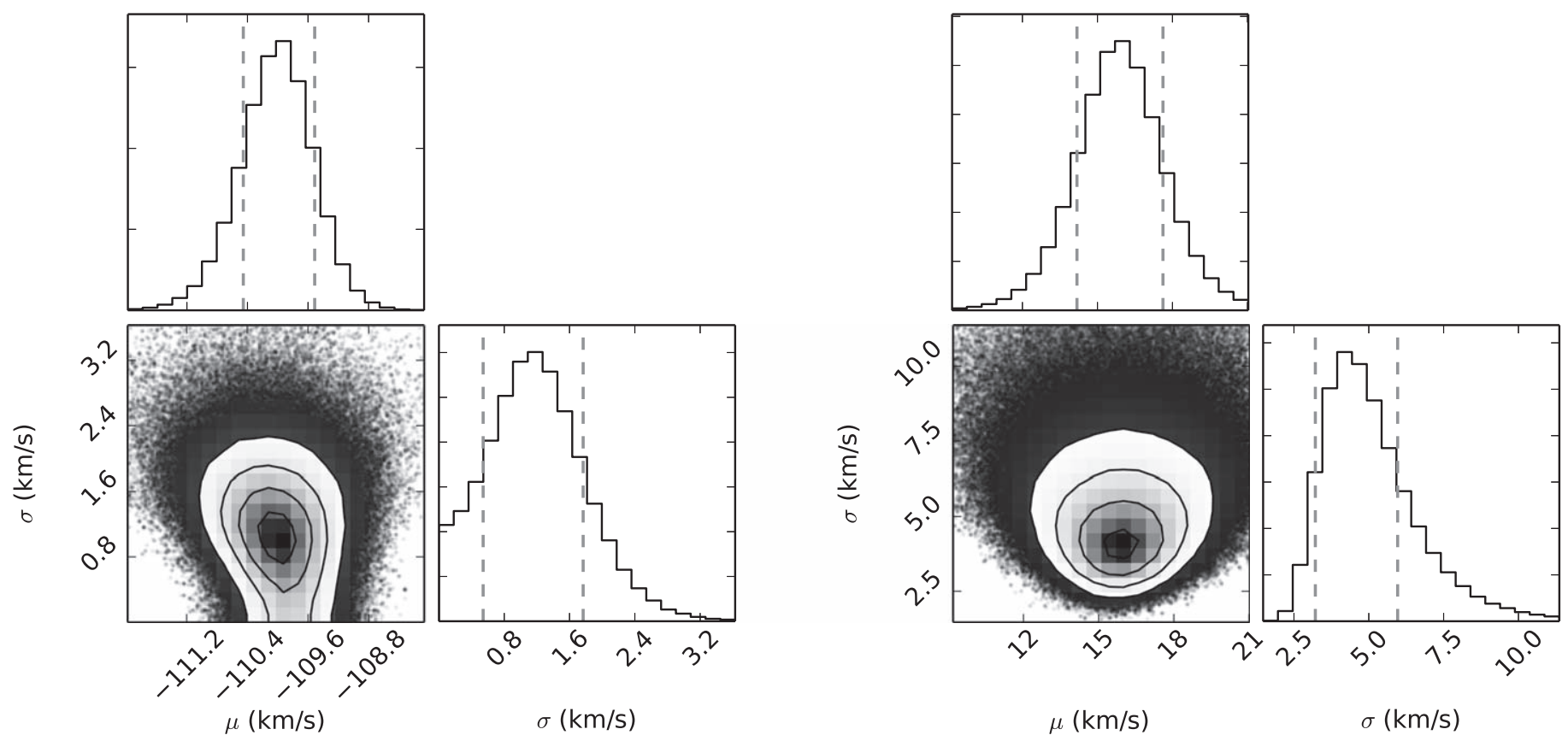

Figure 6. (Left) Corner plot for the velocity and velocity dispersion of Gru II. The upper limit on the velocity dispersion is $2.0 \mathrm{~km} \mathrm{~s} \mathrm{~s}^{-1}$. (Right) Corner plot for the velocity and velocity dispersion of Tuc IV.

consider an LMC whose gravitational field matches the previous Hernquist profile beyond $8.7 \mathrm{kpc}$ but has a flat rotation curve at $91.7 \mathrm{~km} \mathrm{~s}^{-1}$ within $8.7 \mathrm{kpc}$, in approximate agreement with a variety of kinematic data for the LMC. We find that this change only affects the probability of Tuc IV being an LMC satellite at the $\sim 1 \%$ level and thus does not influence our conclusions.

Interestingly, even though we have assigned Tuc IV a modest probability of being a Magellanic satellite, its present-day position and velocity relative to the LMC are comparable to those of the
Magellanic satellites in Erkal \& Belokurov (2019). Figure 9 shows the position and velocity relative to the LMC for the satellites considered in Erkal \& Belokurov (2019) and Tuc IV. Tuc IV sits in a location in this space similar to the Magellanic satellites.

Given that Tuc IV has passed directly through the LMC in the recent past, we next evaluate the tidal shock it experienced from the LMC. At a radius of $4.1 \mathrm{kpc}$, the LMC has a circular velocity of $\sim 90 \mathrm{~km} \mathrm{~s}^{-1}$ (Olsen et al. 2011; van der Marel \& Kallivayalil 2014; Vasiliev 2018). The corresponding enclosed mass is $7.7 \times 10^{9} M_{\odot}$. Naively taking the mass within the half- 


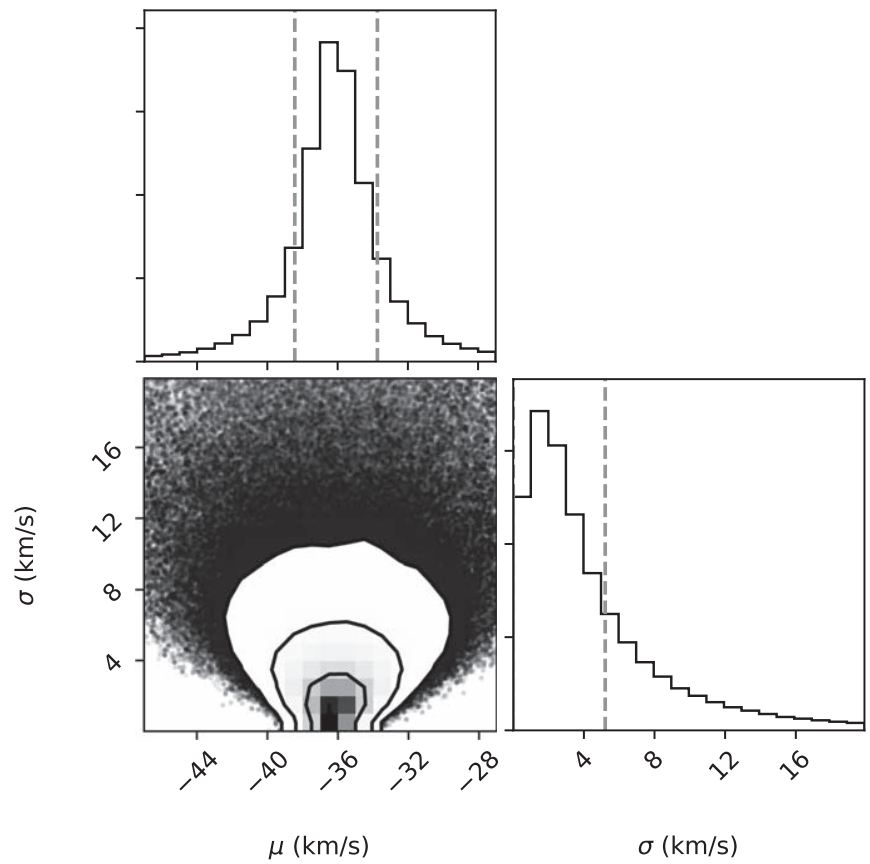

Figure 7. Corner plot for the velocity and velocity dispersion of Tuc V.

light radius of Tuc IV from Section 4.2, the Jacobi radius of Tuc IV at the time of minimum distance to the LMC would be $188 \mathrm{pc}, \sim 1.5$ times its present half-light radius. If much of the dark matter halo of Tuc IV had previously been stripped by encounters with either the Magellanic Clouds or the Milky Way, we would expect this close passage to significantly disturb its internal structure. ${ }^{49}$ In particular, if the true (undisturbed) velocity dispersion of Tuc IV is less than $2.4 \mathrm{~km} \mathrm{~s}^{-1}$, then the tidal radius of Tuc IV during the closest approach to the LMC would be within its half-light radius, implying a major distortion that would likely affect the observed kinematics. However, if Tuc IV retained a massive, extended dark matter halo until this recent event, then its tidal radius probably remained at $\gtrsim 3 r_{1 / 2}$, insulating its stars from the tidal influence of the LMC. It is therefore possible in principle for Tuc IV to have survived this interaction without major damage to its stellar component. We note that several crossing times of Tuc IV have elapsed since the collision, so the system could have reached a new equilibrium even if it was significantly perturbed by the encounter. The uncertainties on the impact parameter, the inner mass distribution of the LMC, and the dynamical mass of Tuc IV are large enough that stronger conclusions would require much more detailed calculations and $N$-body simulations.

\section{Discussion}

One of the primary goals of this study is to determine the nature of Gru II, Tuc IV, and Tuc V. Ideally, satellite classifications are based on direct (a dynamical mass significantly larger than the stellar mass) or indirect (retention of supernova ejecta) evidence for the presence of dark matter (Willman \& Strader 2012). However, as fainter and fainter systems are studied, the detection of nonzero velocity and/or metallicity dispersions becomes more and more difficult (e.g.,

\footnotetext{
${ }^{49}$ It may also be interesting to consider whether the impact of Tuc IV left observable imprints on the disk of the LMC.
}

Martin et al. 2016; Kirby et al. 2017; Simon et al. 2017; Longeard et al. 2018; Ji et al. 2019).

Even without spectroscopy, the large physical sizes $\left(r_{\text {half }}>90 \mathrm{pc}\right)$ of Gru II and Tuc IV strongly suggest that they are galaxies. While outer halo globular clusters can have half-light radii of $\sim 20 \mathrm{pc}$, and in extreme cases tidal shocking of clusters at their orbital pericenter can briefly lead to sizes as large as $\sim 40$ pc (e.g., Contenta et al. 2017), there are no known processes that will inflate the actual or apparent radius of a cluster to $\sim 100 \mathrm{pc}$. The observed internal kinematics of Tuc IV are consistent with this classification, with a mass-to-light ratio within its half-light radius of $\sim 3100 M_{\odot} / L_{\odot}$. We therefore consider Tuc IV to be a spectroscopically confirmed dwarf galaxy. For Gru II, we are not able to detect spectroscopically any evidence for dark matter, although the measurements certainly do not rule out a substantial dark matter content either. The mean metallicity of Gru II is similar to or just below those of the most metal-poor known globular clusters (e.g., Sobeck et al. 2011; Simpson 2018), providing some support for the idea that it is a dwarf. Combining that information with its size, we conclude that Gru II is very likely a galaxy, although more direct evidence would still be desirable.

For Tuc V, we cannot rely on any of the above arguments as we lack a resolved velocity or metallicity dispersion. However, its revised half-light radius of 34 pc (Section 4.1) is now more consistent with a dwarf galaxy classification, as it (at least slightly) exceeds the size of known clusters. The mean metallicity of $[\mathrm{Fe} / \mathrm{H}]=-2.17$ is $\sim 0.5 \mathrm{dex}$ higher than would be expected for a dwarf of its luminosity according to the luminosity-metallicity relation of Kirby et al. (2013b), but because this measurement is based on just two stars, the significance of the discrepancy is not large. Only a small fraction of the Galactic globular cluster population is located at distances comparable to that of Tuc V, but among the clusters in the outer halo, the orbital properties of Tuc V would not stand out (e.g., Baumgardt et al. 2019). Based on the available data, we regard the nature of Tuc $\mathrm{V}$ as undetermined until further spectroscopic observations are obtained.

Conn et al. (2018) obtained Gemini/GMOS-S imaging of Tuc $\mathrm{V}$ extending $\sim 3$ mag deeper than the DES imaging in which Tuc V was discovered. They clearly detected the stellar population identified as Tuc V by Drlica-Wagner et al. (2015), but based on the apparent irregularity of the spatial distribution of those stars, they concluded that Tuc V is either a chance grouping of stars in the SMC, a structure in the SMC halo, or a tidally disrupted star cluster rather than a bound stellar system. We disagree with their interpretation of Tuc V. Our spectroscopy shows that the mean velocity of Tuc $\mathrm{V}$ is offset from that of the SMC by nearly $200 \mathrm{~km} \mathrm{~s}^{-1}$, ruling out the hypothesis that Tuc $\mathrm{V}$ is an overdensity (either real or a line-of-sight projection) associated with the SMC. As mentioned above, the spectroscopic measurements cannot currently distinguish between a dwarf galaxy and a globular cluster, but in either case there is no significant evidence favoring tidal disruption. Analyses by Martin et al. (2008), Walsh et al. (2008), and Muñoz et al. (2010) have shown that irregular low surface brightness features around ultra-faint satellites such as those seen in the vicinity of Tuc $\mathrm{V}$ are generally not statistically significant, and instead are consistent with being the result of Poisson fluctuations from drawing a small number of stars from a smooth spatial distribution. Photometric uncertainties and star-galaxy contamination at faint magnitudes can also 

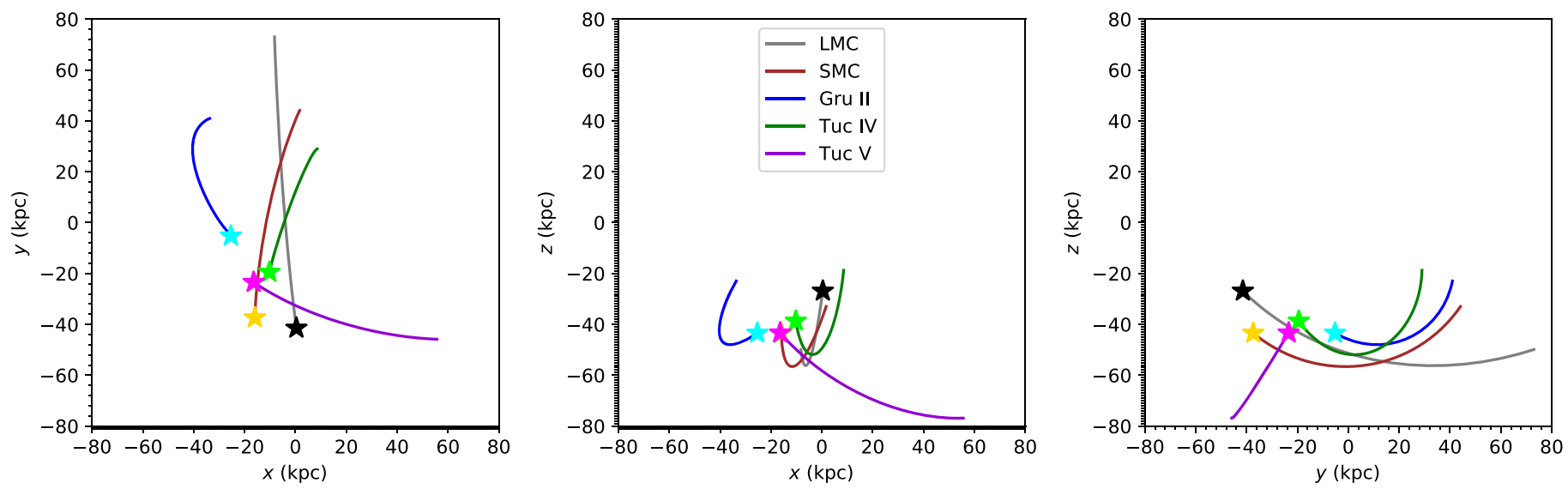

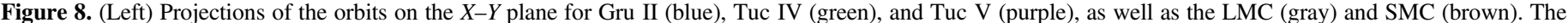

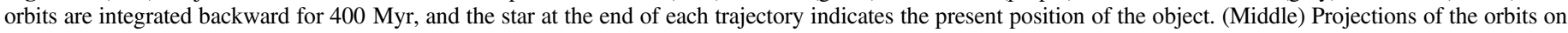

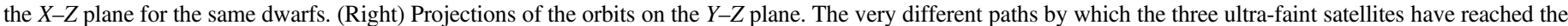
current apparent grouping are clear. The LMC and Tuc IV orbits intersect $\sim 150$ Myr ago in all three planes.

contribute to the apparent presence of tidal debris in groundbased imaging (Mutlu-Pakdil et al. 2019). Based on the revised size of Tuc V determined in Section 4.1, we suggest that the small GMOS field of view $(5.5 \times 5.5)$ hinders a robust structural analysis of the system. In particular, Muñoz et al. (2012) showed that accurately determining the half-light radius of a low surface brightness stellar system requires imaging over a field of view $>3 \times$ the half-light radius. For the previously published size of Tuc V, GMOS meets this criterion, but with our new measurement it does not.

\subsection{J-factor}

The Milky Way's dwarf galaxies are among the best targets for searches for dark matter annihilation and decay radiation because they have high dark matter densities, are located close to the Sun, and are nearly background free. Analyses of $\gamma$-rays from the LAT instrument on the Fermi telescope probe the thermal cross section (Ackermann et al. 2015; Albert et al. 2017). The calculation of the predicted dark matter flux is split into two components. The first is dependent on the distribution of dark matter within the dwarf (the astrophysics component) and the second is related to properties of the dark matter particle(s), such as the cross sections or mass (particle physics component). Adding new dwarf galaxies to the searches improves the reach in dark matter parameter space.

The astrophysics components of the calculation mentioned above are commonly referred to as the $\mathrm{J}$-factor and D-factor for dark matter annihilation and decay, respectively. The J-factor is the integral over the line of sight of the square of the dark matter density, $J(\theta)=\int \rho_{\mathrm{DM}}^{2} d \Omega d l$, and the D-factor is the linear analog, $D(\theta)=\int \rho_{\mathrm{DM}} d \Omega d l$. The standard approach to measuring $\rho_{\mathrm{DM}}$ is to solve the spherical Jeans equation using the observed line-of-sight velocity dispersion (e.g., Bonnivard et al. 2015; Strigari 2018).

Briefly, we solve the spherical Jeans equations, project the velocity dispersion into the line-of-sight direction, and compare the stellar velocity data to the model predictions to determine dark matter parameter distributions. We assume the stellar distribution follows a Plummer (1911) profile, the dark matter density profile follows a Navarro-Frenk-White profile (Navarro et al. 1996), and the stellar anisotropy is constant with radius. We treat the distance, ellipticity, and stellar half- light radius as free parameters with Gaussian priors to account for their measurement errors. This framework is similar in scope to most J-factor analyses for Milky Way dwarfs (e.g., Strigari et al. 2008; Bonnivard et al. 2015). For additional details, see Pace \& Strigari (2019).

Given the upper limit on the velocity dispersion for Gru II, we are similarly only able to set an upper limit on the J-factor (see Table 4). We also find $\log _{10} D<16.6,17.2$ at solid angles of $0.2,0.5$. For Tuc IV, we calculate integrated J-factors of $\log _{10} J=18.0 \pm 0.6,18.2_{-0.5}^{+0.6}, 18.4_{-0.5}^{+0.6}$ within solid angles of $\theta=0.1,0.2,0.5$ in logarithmic units of $\mathrm{GeV}^{2} \mathrm{~cm}^{-5}$ (i.e., $\log _{10}\left(J(\theta) / \mathrm{GeV}^{2} \mathrm{~cm}^{-5}\right)$ ) and integrated D-factors of $\log _{10} D=17.0 \pm 0.3,17.5 \pm 0.3,18.0 \pm 0.4$ within the same solid angles in logarithmic units of $\mathrm{GeV} \mathrm{cm}^{-2}$ (see Table 5). Upper limits on the J-factor of Tuc $\mathrm{V}$ are listed in Table 6. We also set upper limits of $\log _{10} D<18.0,18.6$ at solid angles of $0.2,0.5$ for Tuc V. Because of the small sample size for Tuc V, the upper limits are not very meaningful, whereas Gru II has one of the smallest J-factors given the computed upper limit. The J-factor for Tuc IV is not very large compared to other ultra-faint dwarfs mostly due to its large size, but agrees with scaling relations based on its velocity dispersion, distance, and half-light radius (Pace \& Strigari 2019).

Using the sizes, distances, and luminosities of dwarfs with measured kinematics, Pace \& Strigari (2019) made predictions for dwarfs lacking stellar kinematics. They predicted $\log J=18.4,18.1,18.9$ for Gru II, Tuc IV, and Tuc V, respectively. The prediction for Tuc IV agrees with our J-factor measurement whereas the prediction for Gru II is much larger than our upper limit, emphasizing the importance of stellar kinematics for determining accurate J-factors.

Tuc IV is one of the four dwarfs with an excess $(\sim 2 \sigma)$ of gamma-rays detected by Fermi (Albert et al. 2017). Two of the other dwarfs with an excess, Ret II and Tuc III, have large and small J-factors, respectively, relative to the ultra-faint population as a whole (Pace \& Strigari 2019). The Tuc IV J-factor falls between these two. Albert et al. (2017) noted that there was no correlation between the measured (or predicted) J-factor and the flux upper limit for the dwarf galaxy population, and we find that Tuc IV continues this trend. With a measured J-factor, Tuc IV will be a useful addition to any future searches of dark matter annihilation or decay. 


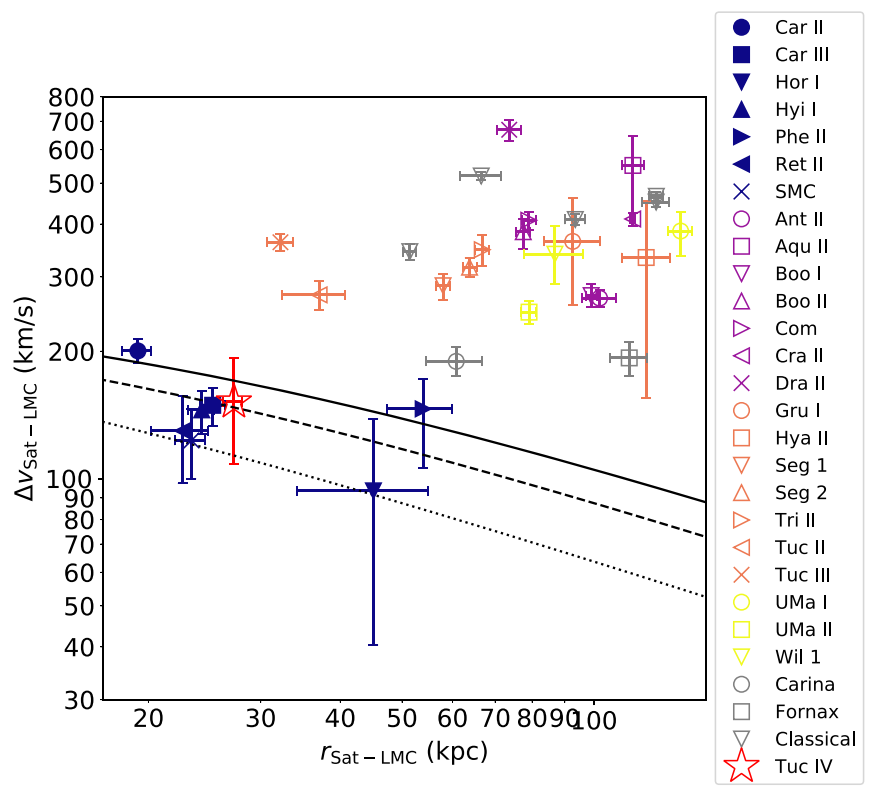

Figure 9. Distance and speed of satellites with respect to the LMC. This figure is almost identical to the one in Erkal \& Belokurov (2019), except that we have also included the relative distance and velocity of Tuc IV. The dotted, dashed, and solid lines show the escape velocity for an LMC with masses of 5, 10, $15 \times 10^{10} M_{\odot}$ and scale radii of $6.2,12.4,17.1 \mathrm{kpc}$, respectively. The solid blue markers show the satellites that Erkal \& Belokurov (2019) identified as Magellanic satellites. Interestingly, Tuc IV sits at a similar position in phase space.

\subsection{Assessing the Possibility of Tidal Stripping}

Since their discovery $15 \mathrm{yr}$ ago, extensive speculation has centered on the effects of tides on the ultra-faint dwarfs (e.g., Zucker et al. 2006a; Coleman et al. 2007; Peñarrubia et al. 2008; Muñoz et al. 2010; Bovill \& Ricotti 2011; Deason et al. 2012; Łokas et al. 2012; Collins et al. 2017; Mutlu-Pakdil et al. 2019). Tidal stripping is particularly important for interpreting the masses of these systems; a galaxy that has recently experienced a tidal shock may have a velocity dispersion that is not representative of its current mass (e.g., Küpper et al. 2017), and over many orbits, stripping can potentially reduce the mass of a galaxy by an order of magnitude or more (e.g., Kravtsov et al. 2004; Peñarrubia et al. 2008). Tidal stripping may also have significant implications for the number of satellite galaxies and their radial distribution relative to the Milky Way (e.g., Garrison-Kimmel et al. 2017) as well as for the luminosity-metallicity relationship in the dwarf galaxy regime (e.g., Kirby et al. 2013b).

For individual systems, the minimum observed velocity dispersion for dwarf galaxies has been decreasing with new discoveries and improved kinematics (e.g., Collins et al. 2017; Koposov et al. 2018), and the previous examples of systems with unusually cold internal kinematics for their luminosities (Kirby et al. 2013a; Caldwell et al. 2017; Simon et al. 2017) all have substantial evidence for tidal stripping. In particular, Segue $2\left(\sigma<2.2 \mathrm{~km} \mathrm{~s}^{-1}\right.$; Kirby et al. 2013a; Simon 2019) is at least 0.6 dex more metal-rich than would be expected from its luminosity, Tucana III ( $\sigma<1.2 \mathrm{~km} \mathrm{~s}^{-1}$; Simon et al. 2017) exhibits tidal tails (Drlica-Wagner et al. 2015; Li et al. 2018a), and the large radius of Crater II $\left(\sigma=2.7 \pm 0.3 \mathrm{~km} \mathrm{~s}^{-1}\right.$; Caldwell et al. 2017) implies significant tidal mass loss on its derived orbit (Sanders et al. 2018; Fu et al. 2019). Gru II does not obviously fit this trend, with an upper limit to its velocity dispersion comparable to that for Tuc III and Segue 2 but no detected tidal tails, ${ }^{50}$ as well as a metallicity that is compatible with the dwarf galaxy luminosity-metallicity relationship at its present luminosity. However, using the $1.6 \times 10^{12} M_{\odot}$ Milky Way mass model from Carlin \& Sand (2018) and assuming that (1) the tidal radius is equal to the Jacobi radius as defined by Binney \& Tremaine (2008) and (2) no mass is present beyond the half-light radius, the tidal radius of Gru II at its current location is $296\left(\sigma / 2.0 \mathrm{~km} \mathrm{~s}^{-1}\right)^{2 / 3} \mathrm{pc}$. Thus, even if the true velocity dispersion is equal to the $2 \sigma$ upper limit determined in Section $4.2, r_{\text {tidal }} / r_{1 / 2}=3.1$, implying that up to $\sim 10 \%$ of the stars in Gru II could be vulnerable to stripping. ${ }^{51}$ If the actual velocity dispersion is significantly smaller, even more stars could be stripped, especially as Gru II approaches the pericenter of its orbit.

Under the same conservative assumptions as for Gru II above, we calculate a tidal radius for Tuc IV of $481 \mathrm{pc}$ $\left(3.8 r_{1 / 2}\right)$. Thus, even if Tuc IV lacks an extended dark matter halo, very few of its stars are beyond its tidal radius and likely to be tidally stripped by the Milky Way. As discussed in Section 4.4.2, whether the central portion of Tuc IV remained tightly bound during its recent collision with the LMC depends on whether the system retained a massive halo until that time. Even if so, it is certainly possible that the outer regions of the dwarf, as well as much of its dark matter, could have been removed by that interaction.

For Tuc V, we find a tidal radius of $386\left(\sigma / 7.4 \mathrm{~km} \mathrm{~s}^{-1}\right)^{2 / 3}$ pc. The compact size of Tuc $\mathrm{V}$ makes it more resilient to tidal stripping. Unless its velocity dispersion is less than $1.0 \mathrm{~km} \mathrm{~s}^{-1}$, its tidal radius is at least $3 r_{1 / 2}$. Our photometric analysis of Tuc $\mathrm{V}$ determined an elongated shape with an ellipticity of $e=0.51_{-0.18}^{+0.09}$. Some authors have suggested that such shapes are a signature of tidal stripping (e.g., Coleman et al. 2007; Martin et al. 2008; Deason et al. 2012), but more detailed calculations indicate that tidal disruption generally does not induce significant ellipticities (Muñoz et al. 2008). Because its orbit has likely not brought it within $\sim 30 \mathrm{kpc}$ of the Milky Way, we conclude that Tuc V has probably not suffered any tidal stripping.

\subsection{Magellanic Association}

A number of authors have recently examined the question of which nearby dwarf galaxies might actually be satellites of the Magellanic Clouds (Jethwa et al. 2016; Sales et al. 2017; Kallivayalil et al. 2018; Simon 2018; Erkal \& Belokurov 2019; Pardy et al. 2019). In particular, Jethwa et al. (2016), Sales et al. (2017), and Kallivayalil et al. (2018) provided predictions for the kinematics of our targets if they are LMC or SMC satellites. While the radial velocities we measure fall within the $68 \%$ confidence intervals computed by Jethwa et al. (2016) for all three objects, as already noted by Pace \& Li (2019), the proper motions do not agree with the Jethwa et al. predictions. We find that the radial velocity and proper motion in the Galactic longitude direction of Tuc $\mathrm{V}$ both strongly disagree

\footnotetext{
50 Note that Tuc III is on an extremely radial orbit, which will result in more prominent tails than a comparable satellite on a less eccentric orbit.

51 Our assumption here that the dynamical mass is equal to the mass within the half-light radius is of course extremely conservative. In principle, the total mass of the system could be much larger if Gru II is still embedded in an extended dark matter halo, but we have no way of measuring that mass. The calculations here demonstrate that tidal stripping is possible but are not sufficient to establish that it is definitely occurring.
} 
with the predictions of Sales et al. (2017), reinforcing our conclusion from Section 4.4.2 that it is not a Magellanic satellite. For Tuc IV, the observed radial velocity and proper motion in Galactic latitude are in reasonable agreement with Sales et al. (2017), but the proper motion in Galactic longitude differs by $\sim 200 \mathrm{~km} \mathrm{~s}^{-1}$. Given the recent interaction between Tuc IV and the LMC, our calculations in Section 4.4.2 represent a more complete assessment of its membership in the Magellanic group. Sales et al. (2017) do not provide predictions for Gru II because they judge it to have too low a probability of association with the Magellanic Clouds. Relative to the predictions of Kallivayalil et al. (2018), our measurements for Gru II are the closest to matching, but none of our targets have motions that agree with the predicted values in all three dimensions.

\subsection{A Tucana Group?}

As mentioned in Section 1, the satellites Tuc II, Tuc IV, and Tuc V are quite close together, and could potentially be the remnant of a dwarf galaxy group (Drlica-Wagner et al. 2015). However, the radial velocities and proper motions of Tuc IV and Tuc $\mathrm{V}$ place them on quite different orbits from each other, as well as from Tuc II. There is therefore no evident connection between the three satellites, and their current proximity is largely coincidental, as is the case for the close pair of Car II and Car III. It is possible, though, that the gravitational influence of the LMC has played a role in bringing these systems closer together.

\subsection{Connection to Other Halo Substructures}

Koposov et al. (2019) used Gaia DR2 data to trace the Orphan Stream (Belokurov et al. 2007) far into the southern hemisphere, finding that it passes very close to Gru II. At the point of closest approach, the angular separation between the two is $\sim 1^{\circ}$, and the proper motion of the stream stars is also very similar to that of Gru II. However, the accompanying modeling by Erkal et al. (2019) determines a heliocentric velocity for the stream of $\sim-200 \mathrm{~km} \mathrm{~s}^{-1}$ at this position, which is offset by $\sim 90 \mathrm{~km} \mathrm{~s}^{-1}$ from the velocity we measure for Gru II. Moreover, the stream stars are $\sim 10 \mathrm{kpc}$ closer than Gru II (Koposov et al. 2019). The difference in distance and velocity between Gru II and the Orphan Stream likely rules out any connection between the two (Martínez-Vázquez et al. 2019). In our spectroscopic data set, we find two stars close to the expected velocity and proper motion of the stream, DES J220441.57-462244.8 and DES J220458.78-462710.6. Both stars have colors and magnitudes consistent with being RGB stars at a distance modulus $\sim 0.4$ mag smaller than that of Gru II and $\mathrm{CaT}$ metallicities of $[\mathrm{Fe} / \mathrm{H}] \approx-2$ assuming that distance. These stars can be added to the sample of spectroscopic members of the Orphan Stream being assembled by the $\mathrm{S}^{5}$ collaboration ( $\mathrm{Li}$ et al. 2019).

\section{Summary and Conclusions}

We have presented the first spectroscopic analysis of the Milky Way satellites Gru II, Tuc IV, and Tuc V. Using medium-resolution Magellan/IMACS spectroscopy, we identified 21 member stars in Gru II, 11 in Tuc IV, and 3 in Tuc V. We used these data to measure the radial velocity, mean metallicity, and proper motion of each system. We determined the velocity dispersion of Tuc IV, but were only able to derive upper limits on the velocity dispersions of Gru II and Tuc V. None of the three objects have a detectable metallicity spread in the existing data.

Based on its low metallicity and large size, we conclude that Gru II is most likely a dwarf galaxy. The same characteristics for Tuc IV, along with the large dynamical mass indicated by its velocity dispersion, identify it as a dwarf galaxy as well. Because of the small number of bright member stars in Tuc V, our constraints on its velocity dispersion, mean metallicity, and metallicity dispersion are very weak. Combined with its small size, we are unable to draw any significant conclusions about whether Tuc V is a dwarf galaxy or globular cluster.

We employed the three-dimensional velocities of the three satellites to compute their orbits around the Milky Way. Gru II and Tuc IV are on eccentric orbits with pericenters of $\sim 20-30$ $\mathrm{kpc}$ and apocenters of $\sim 50-60 \mathrm{kpc}$, similar to the orbits of other nearby ultra-faint dwarfs (e.g., Fritz et al. 2018; Simon 2018). In contrast, the orbit of Tuc V likely extends beyond a distance of $100 \mathrm{kpc}$, and there is a nonnegligible chance that Tuc V is on its first infall to the Milky Way. All three systems are currently approaching pericenter. By projecting the orbits backward in time, we discovered that Tuc IV recently collided with the LMC, with an impact parameter of $\sim 4 \mathrm{kpc}$. Based on their orbits and internal kinematics, we conclude that Gru II could have suffered modest tidal stripping by the Milky Way, Tuc IV could have been stripped by the LMC, and Tuc V is unlikely to have been stripped.

This publication is based upon work supported by the National Science Foundation under grant AST-1714873. J.D.S. was also partially supported by program number HST-GO14734, provided by NASA through a grant from the Space Telescope Science Institute, which is operated by the Association of Universities for Research in Astronomy, Incorporated, under NASA contract NAS5-26555. Some of this work was carried out during a stay at the Kavli Institute for Theoretical Physics, which is supported in part by the National Science Foundation under grant No. NSF PHY-1748958, for the program The Small-Scale Structure of Cold(?) Dark Matter. T.S.L. was supported by NASA through Hubble Fellowship grant HST-HF2-51439.001 awarded by the Space Telescope Science Institute, which is operated by the Association of Universities for Research in Astronomy, Inc., for NASA, under contract NAS5-26555. We thank Dan Kelson for many helpful conversations regarding IMACS data reduction and Petchara Pattarakijwanich for early contributions to the IMACS reduction procedures, as well as the anonymous referee for comments that helped improve the paper. This research has made use of NASA's Astrophysics Data System Bibliographic Services. Contour plots were generated using corner.py (Foreman-Mackey 2016).

Funding for the DES Projects has been provided by the U.S. Department of Energy, the U.S. National Science Foundation, the Ministry of Science and Education of Spain, the Science and Technology Facilities Council of the United Kingdom, the Higher Education Funding Council for England, the National Center for Supercomputing Applications at the University of Illinois at Urbana-Champaign, the Kavli Institute of Cosmological Physics at the University of Chicago, Financiadora de Estudos e Projetos, Fundação Carlos Chagas Filho de Amparo à Pesquisa do Estado do Rio de Janeiro, Conselho Nacional de 
Desenvolvimento Científico e Tecnológico and the Ministério da Ciência e Tecnologia, the Deutsche Forschungsgemeinschaft, and the Collaborating Institutions in the Dark Energy Survey. The DES participants from Spanish institutions are partially supported by MINECO under grants AYA201239559, ESP2013-48274, FPA2013-47986, and Centro de Excelencia Severo Ochoa SEV-2012-0234, some of which include ERDF funds from the European Union. This material is based upon work supported by the National Science Foundation under grant No. 1138766.

The Collaborating Institutions are Argonne National Laboratory, the University of California at Santa Cruz, the University of Cambridge, Centro de Investigaciones Energéticas, Medioambientales y Tecnológicas-Madrid, the University of Chicago, University College London, the DES-Brazil Consortium, the Eidgenössische Technische Hochschule (ETH) Zürich, Fermi National Accelerator Laboratory, the University of Edinburgh, the University of Illinois at Urbana-Champaign, the Institut de Ciències de l'Espai (IEEC/CSIC), the Institut de Física d'Altes Energies, Lawrence Berkeley National Laboratory, the Ludwig-Maximilians Universität and the associated Excellence Cluster Universe, the University of Michigan, the National Optical Astronomy Observatory, the University of Nottingham, The Ohio State University, the University of Pennsylvania, the University of Portsmouth, SLAC National Accelerator Laboratory, Stanford University, the University of Sussex, and Texas A\& M University.

Facility: Magellan:I (IMACS).

\section{ORCID iDs}

T. S. Li (1) https://orcid.org/0000-0002-9110-6163

A. B. Pace (1) https://orcid.org/0000-0002-6021-8760

A. Drlica-Wagner (1) https://orcid.org/0000-0001-8251-933X

J. L. Marshall (1) https://orcid.org/0000-0003-0710-9474

T. Hansen (1) https://orcid.org/0000-0001-6154-8983

K. Kuehn (i) https://orcid.org/0000-0003-0120-0808

C. Lidman (1) https://orcid.org/0000-0003-1731-0497

J. Annis (i) https://orcid.org/0000-0002-0609-3987

D. Brooks (1) https://orcid.org/0000-0002-8458-5047

D. L. Burke (1) https://orcid.org/0000-0003-1866-1950

M. Carrasco Kind (1) https://orcid.org/0000-0002-4802-3194

J. García-Bellido (i) https://orcid.org/0000-0002-9370-8360

D. W. Gerdes (i) https://orcid.org/0000-0001-6942-2736

D. Gruen (1) https://orcid.org/0000-0003-3270-7644

R. A. Gruendl (1) https://orcid.org/0000-0002-4588-6517

D. L. Hollowood (1) https://orcid.org/0000-0002-9369-4157

R. Miquel (i) https://orcid.org/0000-0002-6610-4836

A. Palmese (i) https://orcid.org/0000-0002-6011-0530

F. Paz-Chinchón (1) https://orcid.org/0000-0003-1339-2683

A. Roodman (ib https://orcid.org/0000-0001-5326-3486

E. Sanchez (ib https://orcid.org/0000-0002-9646-8198

M. Smith (i) https://orcid.org/0000-0002-3321-1432

G. Tarle $\mathbb{1}$ h https://orcid.org/0000-0003-1704-0781

A. R. Walker (1) https://orcid.org/0000-0002-7123-8943

\section{References}

Ackermann, M., Albert, A., Anderson, B., et al. 2015, PhRvL, 115, 231301 Albert, A., Anderson, B., Bechtol, K., et al. 2017, ApJ, 834, 110 An, D., Johnson, J. A., Clem, J. L., et al. 2008, ApJS, 179, 326 Battaglia, G., \& Starkenburg, E. 2012, A\&A, 539, A123

Baumgardt, H., Hilker, M., Sollima, A., \& Bellini, A. 2019, MNRAS, 482,5138
Bechtol, K., Drlica-Wagner, A., Balbinot, E., et al. 2015, ApJ, 807, 50 Belokurov, V., Evans, N. W., Irwin, M. J., et al. 2007, ApJ, 658, 337

Binney, J., \& Tremaine, S. 2008, Galactic Dynamics (2nd ed.; Princeton, NJ: Princeton Univ. Press)

Bonnivard, V., Combet, C., Daniel, M., et al. 2015, MNRAS, 453, 849

Bovill, M. S., \& Ricotti, M. 2011, ApJ, 741, 17

Bovy, J. 2015, ApJS, 216, 29

Bressan, A., Marigo, P., Girardi, L., et al. 2012, MNRAS, 427, 127

Caldwell, N., Walker, M. G., Mateo, M., et al. 2017, ApJ, 839, 20

Carlin, J. L., \& Sand, D. J. 2018, ApJ, 865, 7

Carrera, R., Pancino, E., Gallart, C., \& del Pino, A. 2013, MNRAS, 434, 1681

Chubak, C., Marcy, G., Fischer, D. A., et al. 2012, arXiv:1207.6212

Coleman, M. G., de Jong, J. T. A., Martin, N. F., et al. 2007, ApJL, 668, L43

Collins, M. L. M., Tollerud, E. J., Sand, D. J., et al. 2017, MNRAS, 467, 573

Conn, B. C., Jerjen, H., Kim, D., \& Schirmer, M. 2018, ApJ, 852, 68

Contenta, F., Gieles, M., Balbinot, E., \& Collins, M. L. M. 2017, MNRAS, 466, 1741

Cooper, M. C., Newman, J. A., Davis, M., Finkbeiner, D. P., \& Gerke, B. F. 2012, spec2d: DEEP2 DEIMOS Spectral Pipeline, v.1.1.4, Astrophysics Source Code Library, ascl:1203.003

Deason, A. J., Belokurov, V., Evans, N. W., Watkins, L. L., \& Fellhauer, M. 2012, MNRAS, 425, L101

Deason, A. J., Wetzel, A. R., Garrison-Kimmel, S., \& Belokurov, V. 2015, MNRAS, 453, 3568

Dehnen, W., \& Binney, J. 1998, MNRAS, 294, 429

DES Collaboration, Abbott, T. M. C., Abdalla, F. B., et al. 2018, ApJS, 239, 18 Diehl, H. T., Abbott, T. M. C., Annis, J., et al. 2014, Proc. SPIE, 9149, $91490 \mathrm{~V}$ D’Onghia, E., \& Lake, G. 2008, ApJL, 686, L61

Dressler, A., Bigelow, B., Hare, T., et al. 2011, PASP, 123, 288

Dressler, A., Hare, T., Bigelow, B. C., \& Osip, D. J. 2006, Proc. SPIE, 6269, $62690 \mathrm{~F}$

Drlica-Wagner, A., Bechtol, K., Allam, S., et al. 2016, ApJL, 833, L5

Drlica-Wagner, A., Bechtol, K., Rykoff, E. S., et al. 2015, ApJ, 813, 109

Erkal, D., \& Belokurov, V. A. 2019, MNRAS, submitted, arXiv:1907.09484

Erkal, D., Belokurov, V., Laporte, C. F. P., et al. 2019, MNRAS, 487, 2685

Erkal, D., Li, T. S., Koposov, S. E., et al. 2018, MNRAS, 481, 3148

Fillingham, S. P., Cooper, M. C., Kelley, T., et al. 2019, MNRAS, submitted, arXiv: 1906.04180

Flaugher, B., Diehl, H. T., Honscheid, K., et al. 2015, AJ, 150, 150

Foreman-Mackey, D. 2016, JOSS, 1, 24

Foreman-Mackey, D., Hogg, D. W., Lang, D., \& Goodman, J. 2013, PASP, 125,306

Fritz, T. K., Battaglia, G., Pawlowski, M. S., et al. 2018, A\&A, 619, A103

Fu, S. W., Simon, J. D., \& Alarcón Jara, A. G. 2019, ApJ, 883, 11

Gaia Collaboration, Brown, A. G. A., Vallenari, A., et al. 2018, A\&A, 616, A1

Gaia Collaboration, Prusti, T., de Bruijne, J. H. J., et al. 2016, A\&A, 595, A1

Garrison-Kimmel, S., Wetzel, A., Bullock, J. S., et al. 2017, MNRAS, 471, 1709

Geha, M., Brown, T. M., Tumlinson, J., et al. 2013, ApJ, 771, 29

Gontcharov, G. A. 2006, AstL, 32, 759

Graczyk, D., Pietrzyński, G., Thompson, I. B., et al. 2014, ApJ, 780, 59

Harris, J., \& Zaritsky, D. 2006, AJ, 131, 2514

Hernquist, L. 1990, ApJ, 356, 359

Jethwa, P., Erkal, D., \& Belokurov, V. 2016, MNRAS, 461, 2212

Ji, A. P., Simon, J. D., Frebel, A., Venn, K. A., \& Hansen, T. T. 2019, ApJ, 870,83

Kallivayalil, N., Sales, L. V., Zivick, P., et al. 2018, ApJ, 867, 19

Kallivayalil, N., van der Marel, R. P., Besla, G., Anderson, J., \& Alcock, C. 2013, ApJ, 764, 161

Kim, D., \& Jerjen, H. 2015, ApJL, 808, L39

Kim, D., Jerjen, H., Mackey, D., Da Costa, G. S., \& Milone, A. P. 2015, ApJL, 804, L44

Kirby, E. N., Boylan-Kolchin, M., Cohen, J. G., et al. 2013a, ApJ, 770, 16

Kirby, E. N., Cohen, J. G., Guhathakurta, P., et al. 2013b, ApJ, 779, 102

Kirby, E. N., Cohen, J. G., Simon, J. D., et al. 2017, ApJ, 838, 83

Klimentowski, J., Łokas, E. L., Knebe, A., et al. 2010, MNRAS, 402, 1899

Koposov, S. E., Belokurov, V., Li, T. S., et al. 2019, MNRAS, 485, 4726

Koposov, S. E., Belokurov, V., Torrealba, G., \& Evans, N. W. 2015, ApJ, 805,130

Koposov, S. E., Walker, M. G., Belokurov, V., et al. 2018, MNRAS, 479, 5343

Kravtsov, A. V., Gnedin, O. Y., \& Klypin, A. A. 2004, ApJ, 609, 482

Küpper, A. H. W., Johnston, K. V., Mieske, S., Collins, M. L. M., \& Tollerud, E. J. 2017, ApJ, 834, 112

Laevens, B. P. M., Martin, N. F., Bernard, E. J., et al. 2015a, ApJ, 813, 44

Laevens, B. P. M., Martin, N. F., Ibata, R. A., et al. 2015b, ApJL, 802, L18

Li, T. S., Koposov, S. E., Zucker, D. B., et al. 2019, MNRAS, 490, 3508 
Li, T. S., Simon, J. D., Drlica-Wagner, A., et al. 2017, ApJ, 838, 8

Li, T. S., Simon, J. D., Kuehn, K., et al. 2018a, ApJ, 866, 22

Li, T. S., Simon, J. D., Pace, A. B., et al. 2018b, ApJ, 857, 145

Li, Y.-S., \& Helmi, A. 2008, MNRAS, 385, 1365

Łokas, E. L., Kazantzidis, S., \& Mayer, L. 2012, ApJL, 751, L15

Longeard, N., Martin, N., Starkenburg, E., et al. 2018, MNRAS, 480, 2609

Martin, N. F., de Jong, J. T. A., \& Rix, H.-W. 2008, ApJ, 684, 1075

Martin, N. F., Geha, M., Ibata, R. A., et al. 2016, MNRAS, 458, L59

Martínez-Vázquez, C. E., Vivas, A. K., Gurevich, M., et al. 2019, MNRAS, 490, 2183

Massari, D., \& Helmi, A. 2018, A\&A, 620, A155

McMillan, P. J. 2017, MNRAS, 465, 76

Minor, Q. E., Pace, A. B., Marshall, J. L., \& Strigari, L. E. 2019, MNRAS, 487, 2961

Moe, M., Kratter, K. M., \& Badenes, C. 2019, ApJ, 875, 61

Muñoz, R. R., Geha, M., \& Willman, B. 2010, AJ, 140, 138

Muñoz, R. R., Majewski, S. R., \& Johnston, K. V. 2008, ApJ, 679, 346

Muñoz, R. R., Padmanabhan, N., \& Geha, M. 2012, ApJ, 745, 127

Mutlu-Pakdil, B., Sand, D. J., Walker, M. G., et al. 2019, ApJ, 885, 53

Navarro, J. F., Frenk, C. S., \& White, S. D. M. 1996, ApJ, 462, 563

Newman, J. A., Cooper, M. C., Davis, M., et al. 2013, ApJS, 208, 5

Oemler, A., Clardy, K., Kelson, D., Walth, G., \& Villanueva, E. 2017, COSMOS: Carnegie Observatories System for MultiObject Spectroscopy, v.2.21, Astrophysics Source Code Library, ascl:1705.001

Olsen, K. A. G., Zaritsky, D., Blum, R. D., Boyer, M. L., \& Gordon, K. D. 2011, ApJ, 737, 29

Pace, A. B., \& Li, T. S. 2019, ApJ, 875, 77

Pace, A. B., \& Strigari, L. E. 2019, MNRAS, 482, 3480

Pardy, S. A., D'Onghia, E., Navarro, J., et al. 2019, MNRAS, 492, 1543

Peñarrubia, J., Navarro, J. F., \& McConnachie, A. W. 2008, ApJ, 673, 226

Pietrzyński, G., Graczyk, D., Gieren, W., et al. 2013, Natur, 495, 76

Plummer, H. C. 1911, MNRAS, 71, 460

Rodriguez Wimberly, M. K., Cooper, M. C., Fillingham, S. P., et al. 2019, MNRAS, 483, 4031
Sales, L. V., Navarro, J. F., Cooper, A. P., et al. 2011, MNRAS, 418, 648 Sales, L. V., Navarro, J. F., Kallivayalil, N., \& Frenk, C. S. 2017, MNRAS, 465,1879

Sanders, J. L., Evans, N. W., \& Dehnen, W. 2018, MNRAS, 478, 3879

Sharp, R., Saunders, W., Smith, G., et al. 2006, Proc. SPIE, 6269, 62690G

Simon, J. D. 2018, ApJ, 863, 89

Simon, J. D. 2019, ARA\&A, 57, 375

Simon, J. D., Drlica-Wagner, A., Li, T. S., et al. 2015, ApJ, 808, 95

Simon, J. D., \& Geha, M. 2007, ApJ, 670, 313

Simon, J. D., Li, T. S., Drlica-Wagner, A., et al. 2017, ApJ, 838, 11

Simpson, J. D. 2018, MNRAS, 477, 4565

Sobeck, J. S., Kraft, R. P., Sneden, C., et al. 2011, AJ, 141, 175

Spencer, M. E., Mateo, M., Olszewski, E. W., et al. 2018, AJ, 156, 257

Strigari, L. E. 2018, RPPh, 81, 056901

Strigari, L. E., Koushiappas, S. M., Bullock, J. S., et al. 2008, ApJ, 678, 614

Torrealba, G., Belokurov, V., Koposov, S. E., et al. 2018, MNRAS, 475, 5085

Torrealba, G., Koposov, S. E., Belokurov, V., et al. 2016a, MNRAS, 463, 712

Torrealba, G., Koposov, S. E., Belokurov, V., \& Irwin, M. 2016b, MNRAS, 459,2370

van der Marel, R. P., Alves, D. R., Hardy, E., \& Suntzeff, N. B. 2002, AJ, 124, 2639

van der Marel, R. P., \& Kallivayalil, N. 2014, ApJ, 781, 121

Vasiliev, E. 2018, MNRAS, 481, L100

Walker, M. G., Mateo, M., Olszewski, E. W., et al. 2016, ApJ, 819, 53

Walsh, S. M., Willman, B., Sand, D., et al. 2008, ApJ, 688, 245

Watkins, L. L., van der Marel, R. P., Sohn, S. T., \& Evans, N. W. 2019, ApJ, 873,118

Wetzel, A. R., Tollerud, E. J., \& Weisz, D. R. 2015, ApJL, 808, L27

Willman, B., \& Strader, J. 2012, AJ, 144, 76

Wolf, J., Martinez, G. D., Bullock, J. S., et al. 2010, MNRAS, 406, 1220

Yoon, J., Whitten, D. D., Beers, T. C., Lee, Y. S., \& Placco, V. M. 2019, ApJ, submitted, arXiv: 1910.10038

Zucker, D. B., Belokurov, V., Evans, N. W., et al. 2006a, ApJL, 650, L41

Zucker, D. B., Belokurov, V., Evans, N. W., et al. 2006b, ApJL, 643, L103 